ARTICLE

https://doi.org/10.1038/s41467-019-09492-4

\title{
The cyclooxygenase-1/mPGES-1/endothelial prostaglandin EP4 receptor pathway constrains myocardial ischemia-reperfusion injury
}

\author{
Liyuan Zhu' ${ }^{1,8}$, Chuansheng Xu ${ }^{1,8}$, Xingyu Huo ${ }^{1,8}$, Huifeng Hao ${ }^{1}$, Qing Wan ${ }^{1}$, Hong Chen ${ }^{1}$, Xu Zhang ${ }^{2}$,
} Richard M. Breyer ${ }^{3}$, Yu Huang ${ }^{4}$, Xuetao Cao (1) ${ }^{5}$, De-Pei Liu ${ }^{5}$, Garret A. FitzGerald ${ }^{6}$ \& Miao Wang (1) ${ }^{1,7}$

The use of nonsteroidal anti-inflammatory drugs that inhibit cyclooxygenase (COX)- 1 and COX-2, increases heart failure risk. It is unknown whether microsomal $(\mathrm{m})$ prostaglandin (PG) E synthase (S)-1, a target downstream of COX, regulates myocardial (M) ischemia/ reperfusion $(\mathrm{I} / \mathrm{R})$ injury, a key determinant of heart failure. Here we report that $\mathrm{COX}-1$ and mPGES-1 mediate production of substantial amounts of $\mathrm{PGE}_{2}$ and confer cardiac protection in MI/R. Deletion of mPges-1 impairs cardiac microvascular perfusion and increases inflammatory cell infiltration in mouse MI/R. Consistently, mPges-1 deletion depresses the arteriolar dilatory response to $\mathrm{I} / \mathrm{R}$ in vivo and to acetylcholine ex vivo, and enhances leukocyteendothelial cell interaction, which is mediated via PGE receptor-4 (EP4). Furthermore, endothelium-restricted Ep4 deletion impairs microcirculation, and exacerbates $M I / R$ injury, irrespective of EP4 agonism. Treatment with misoprostol, a clinically available PGE analogue, improves microcirculation and reduces MI/R injury. Thus, mPGES-1, a key microcirculation protector, constrains $\mathrm{MI} / \mathrm{R}$ injury and this beneficial effect is partially mediated via endothelial EP4.

\footnotetext{
${ }^{1}$ State Key Laboratory of Cardiovascular Disease, Fuwai Hospital, National Center for Cardiovascular Diseases, Chinese Academy of Medical Sciences and Peking Union Medical College, Beijing 100037, China. ${ }^{2}$ Tianjin Key Laboratory of Metabolic Diseases and Department of Physiology, Tianjin Medical University, Tianjin 300070, China. ${ }^{3}$ Division of Nephrology, Department of Medicine, Vanderbilt University Medical Center, Nashville, TN 37212 , USA ${ }^{4}$ Institute of Vascular Medicine and Li Ka Shing Institute of Health Sciences, The Chinese University of Hong Kong, Hong Kong SAR, China. ${ }^{5}$ Institute of Basic Medical Sciences, Chinese Academy of Medical Sciences and Peking Union Medical College, Beijing 100005, China. ${ }^{6}$ Institute for Translational Medicine and Therapeutics, Perelman School of Medicine, Department of Systems Pharmacology and Translational Therapeutics, University of Pennsylvania, Philadelphia, PA 19104, USA. ${ }^{7}$ Clinical Pharmacology Center, Fuwai Hospital, National Center for Cardiovascular Diseases, Chinese Academy of Medical Sciences and Peking Union Medical College, Beijing 100037, China. ${ }^{8}$ These authors contributed equally: Liyuan Zhu, Chuansheng Xu, Xingyu Huo. Correspondence and requests for materials should be addressed to M.W. (email: miao.wang@pumc.edu.cn)
} 
$\mathrm{N}$ onsteroidal anti-inflammatory drugs (NSAIDs) ameliorate pain and fever by inhibiting cyclooxygenase (COX) activity, thus suppressing biosynthesis of prostanoids. COX (which exists mainly in two isoforms, COX-1 and COX-2) catalyzes the formation of prostaglandin (PG) $\mathrm{H}_{2}$, the common substrate for downstream isomerases to synthesize individual prostanoids, including $\mathrm{PGE}_{2}$ and $\mathrm{PGI}_{2}$. Use of NSAIDs is associated with an increased risk of heart failure ${ }^{1}$. The role of COX pathway in myocardial $(\mathrm{M})$ ischemia/reperfusion (I/R) injury, a key determinant of the subsequent development of heart failure ${ }^{2}$, remains unclear. $\mathrm{MI} / \mathrm{R}$ also constitutes a therapeutic target under intensive investigation ${ }^{2-4}$.

Microsomal (m) PGE synthase (S)-1, the dominant synthetic enzyme for $\mathrm{PGE}_{2}$ production in vivo ${ }^{5}$, is being considered as a new therapeutic target downstream of $\mathrm{COX}^{6-8}$. In contrast to COX-2 inhibitors, deletion of $m P g e s-1$ augments $\mathrm{PGI}_{2}$ production, restraining thrombogenesis and atherogenesis, and modulating the response to vascular injury ${ }^{5,9-12}$. In isolated mouse hearts, both COX-1 and COX-2 mediate recovery of left ventricular developed pressure after ischemia ${ }^{13}$. However, in mice, COX-2 inhibition does not modify infarct size post MI/R ${ }^{14}$, and the role of COX-1 remains undetermined in vivo. Both $\mathrm{PGI}_{2}$ and $\mathrm{PGE}_{2}$ may be formed by COXs in $\mathrm{MI} / \mathrm{R}^{13,15}$. $\mathrm{PGI}_{2}$ restrains $\mathrm{MI} / \mathrm{R}$ injury via its receptor (IP) ${ }^{16}$. In a mouse model of sustained (4 weeks) myocardial infarction, i.e., without reperfusion after coronary occlusion, global mPges- 1 deletion ${ }^{17}$, or its deletion in bone marrow-derived leukocytes ${ }^{18}$, impairs chronic cardiac remodeling. However, mPges-1 deletion does not worsen cardiac ischemic injury after $24 \mathrm{~h}$ coronary occlusion ${ }^{19}$. It remains unknown whether mPGES-1 regulates myocardial I/R injury, a clinically relevant pathologic process in patients with myocardial infarction undergoing reperfusion therapy ${ }^{2}$. $\mathrm{PGE}_{2}$ has four PGE receptors (EP1-4) mediating diverse biological functions ${ }^{20}$. Global deletion of Ep4 increases infarct size post $\mathrm{MI} / \mathrm{R}^{21}$, whereas cardiomyocyte-specific Ep4 deletion reduces cardiac hypertrophy without changing infarct size in a model of sustained $\mathrm{MI}^{22}$, suggesting the potential involvement of cells other than cardiomyocytes in mediating the impact of global Ep4 KO.

Here we report that mPGES-1 protects against acute $M I / R$ injury in mice and this is attributed to its critical role in preserving microcirculation and limiting inflammation in $I / R$. The cardioprotective effect of mPGES-1 is partially mediated through $\mathrm{PGE}_{2}$ action on the endothelial EP4 receptor. Inhibition of COX1/mPGES-1-derived $\mathrm{PGE}_{2}$ contributes to a risk of myocardial injury in the setting of $\mathrm{MI} / \mathrm{R}$.

\section{Results}

COX-1 protects against $M I / R$ injury in mice. COX is the ratelimiting enzyme upstream of mPGES-1. We set out to examine the relative importance of COX-1 and COX-2 in MI/R in vivo. Cox-1 $\mathrm{KO}$ and Cox-2 KO mice and their littermates were subjected to 30-min ischemia by ligating the left anterior descending coronary artery, followed by $24-\mathrm{h}$ reperfusion. The same MI/R protocol was used throughout this study. Deletion of Cox-1, but not Cox-2 significantly increased the percentage of infarct area in the area at risk (AAR), with AAR similar across the groups (Fig. 1a-c). Treatment with celecoxib, a COX-2 selective inhibitor, at $100 \mathrm{mg} / \mathrm{kg}$, a dose previously shown to accelerate experimentally induced thrombosis ${ }^{5}$, did not modify infarct size, whereas Cox-1 deletion increased infarct size after MI/R injury (Fig. 1c-e), confirming a protective role of COX-1 against MI/R injury. Cox-1 deletion suppressed formation of $\mathrm{PGE}_{2}$ (Fig. 1f), a potential mediator of the cardioprotection of COX-1. MI/R increased urinary excretion of $\mathrm{PGE}_{2}$ metabolites, which was mainly produced by COX-1, not COX-2 (Supplementary Fig. 1A, B). Consistently, COX-1 is the more abundant isoform expressed in the heart (Supplementary Fig. 1C), also as previously reported ${ }^{13,23}$.

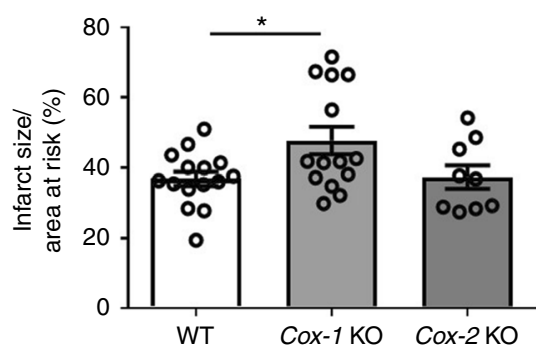

d

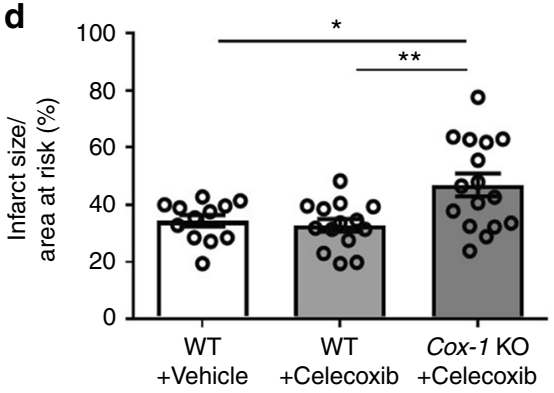

b
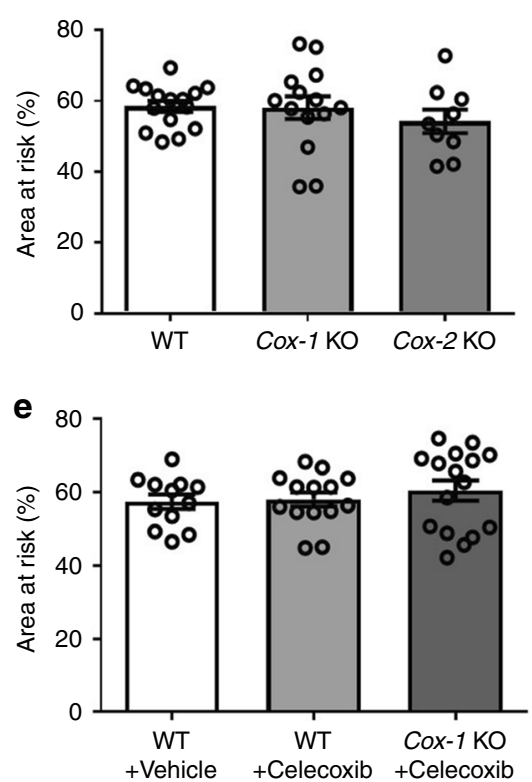

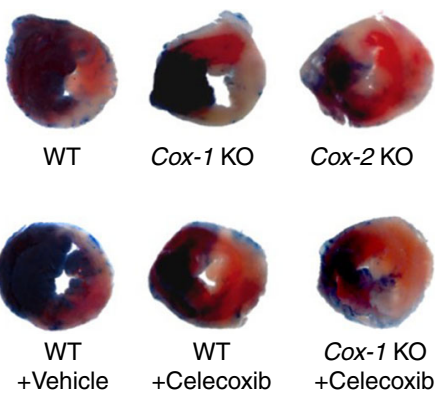

f

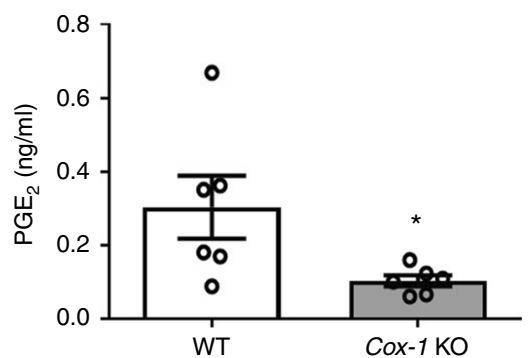

Fig. 1 Impact of Cox-1/2 deletion on MI/R injury. Infarct size (IS) (a) and area at risk (AAR) (b) were quantified $24 \mathrm{~h}$ after I/R injury, as detailed in the Methods. Representative photographs showing TTC stained transverse sections of Evans blue perfused hearts were shown for each study group (c). In a separate study, mice were orally treated with $100 \mathrm{mg} / \mathrm{kg}$ celecoxib or vehicle before and $20 \mathrm{~h}$ after surgery. Representative TTC staining of the heart sections was shown for each group (c), and their infarct size (d) and AAR (e) were quantified. Biosynthesis of PGE 2 (f) was measured by HPLC-MS-MS as detailed in the Methods. One-way ANOVA with Tukey's multiple comparison test (a, $\mathbf{b}, n=15,14,9 ; \mathbf{d}, \mathbf{e}, n=12,14,16$. Individual $n$ numbers in each panel are shown in same order as their corresponding groups from left to right, and this arrangement is applied to other relevant figures in the text.). Unpaired Student's $t$ test $(\mathbf{f}, n=6)$. Statistical significance is demarked as ${ }^{\star} P<0.05,{ }^{\star \star} P<0.01$, and ${ }^{\star \star \star} P<0.001$, throughout the text. Error bar indicates SEM 
mPGES-1 protects against MI/R injury. We next focused on the role of mPGES-1 in MI/R injury. Deletion of mPges-1 increased infarct size (Fig. 2a-c) and reduced fractional shortening and ejection fraction (Fig. 2d-f). Naive mPges-1 KO mice showed no difference in ultrasonographic parameters compared with controls (Supplementary Fig. 2A-D). Again, deletion of mPges-1 suppressed the level of $\mathrm{PGE}_{2}$ (Fig. 2g), suggesting an integrative cardioprotective mechanism of COX-1 and mPGES- 1 in the setting of MI/R. Measurement of urinary metabolites of $\mathrm{PGE}_{2}$ and $\mathrm{PGI}_{2}$ indicated mPGES-1 as a major source of $\mathrm{PGE}_{2}$ and a a
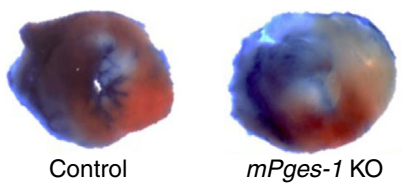

b

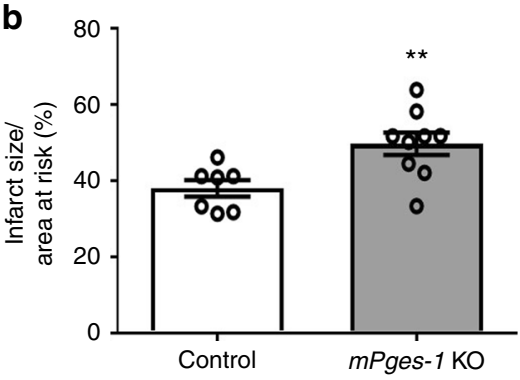

C

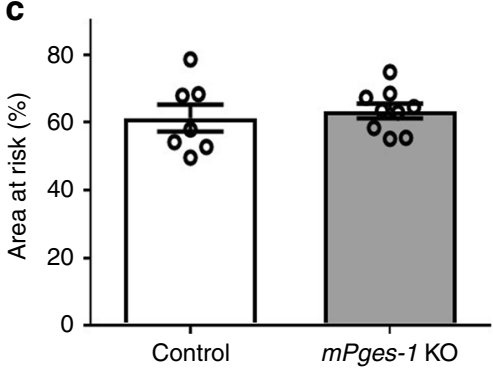

i

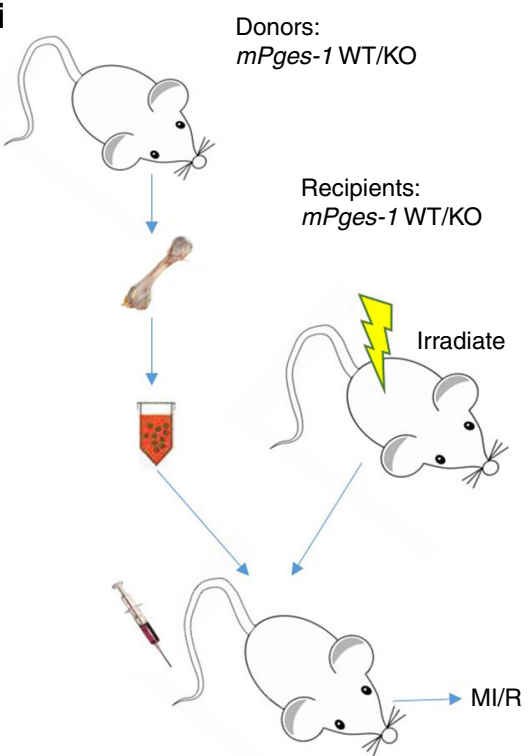

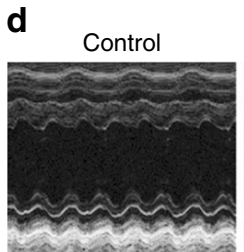

mPges-1 KO
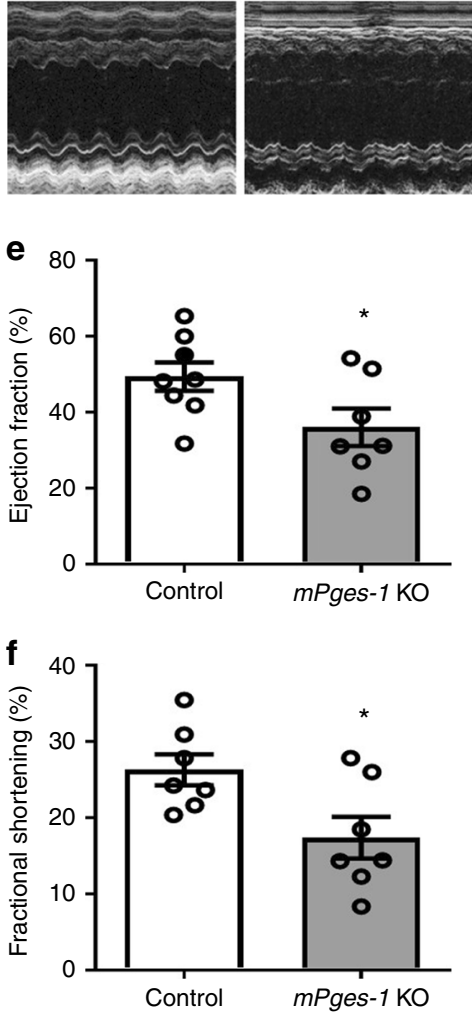

j
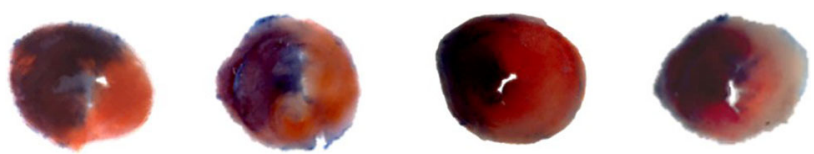

$\begin{array}{ll}\text { Recipient: } & \text { WT } \\ \text { Donor: } & \text { WT }\end{array}$

WT

KO

k

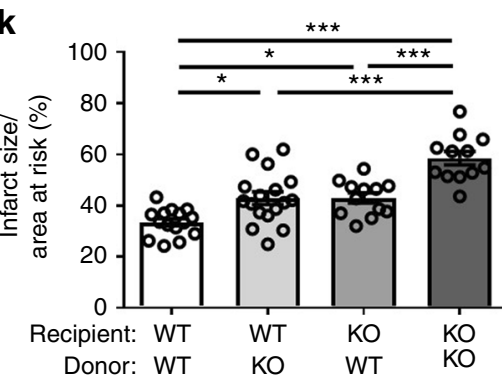

KO

WT

KO

KO

I

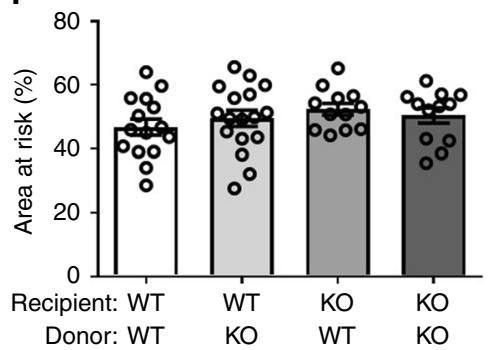

Fig. 2 Impact of mPges-1 deletion on MI/R injury. Mice were subjected to MI/R injury as detailed in the Methods. a Representative photographs of TTC stained sections from Evans blue perfused hearts. Infarct size (b) and AAR (c) were quantified for mPges-1 KO and control mice. Representative echocardiograph (d), ejection fraction (EF) (e), and fractional shortening (FS) (f) were shown. PGE 2 levels were compared (g). Cardiac levels of ATP post MI/R were measured (h). Unpaired Student's $t$ test $(\mathbf{b}, \mathbf{c}, n=7,9 ; \mathbf{e}, n=8,7 ; \mathbf{f}, n=7,7 ; \mathbf{g}, n=8,8 ; \mathbf{h}, n=5)$. Bone marrow cells were transplanted reciprocally between mPges- 1 WT and KO mice (i). The resulting chimeric mice underwent MI/R injury. Representative TTC staining was shown for each group (j). Infarct size (k) and AAR (I) were analyzed with use of one-way ANOVA and Tukey's multiple comparison test $(n=15,17,12,12)$. Error bar indicates SEM 
shunting toward biosynthesis of $\mathrm{PGI}_{2}$ in the face of $\mathrm{PGE}_{2}$ suppression, during MI/R (Supplementary Fig. 2E, F). The role of $\mathrm{PGE}_{2}$ suppression in mediating the deleterious effect of $m$ Pges-1 deletion on $\mathrm{MI} / \mathrm{R}$ injury was further confirmed by treating the animals with misoprostol, a PGE analog, which abolished the exaggerated infarct size due to mPges-1 deletion (Supplementary Fig. 3). Tissue levels of ATP in the area of myocardium at risk after I/R were reduced in mPges- $1 \mathrm{KO}$ mice (Fig. $2 \mathrm{~h}$ ) while the ratio of ADP to ATP was increased (Supplementary Fig. 4A, B). Plasma levels of IL22 were not altered by mPges-1 deletion in MI/ R (Supplementary Fig. 4C).

Reciprocal bone-marrow transplantation was conducted between mPges-1 WT and KO mice (Fig. 2i). Specific deletion of mPges-1 in bone marrow-derived cells (BMCs) or in non$\mathrm{BMCs}$ increased infarct size post MI/R injury, and cardiac injury was further exacerbated by the loss of mPges- 1 in both compartments (Fig. 2j-1). Impaired ejection fraction and fractional shortening were observed in the chimeric mice with $m$ Pges-1 KO in bone marrow or in both compartments, compared with WT to WT chimeric mice (Supplementary Fig. 5A-C). Left ventricular contractile function correlated with infarct size (Supplementary Fig. 5D). Hence, both BMC and non-BMC mediated the protective role of $\mathrm{mPGES}-1$ in restraining infarct size in $\mathrm{MI} / \mathrm{R}$ injury.

mPges-1 deletion impairs microvascular perfusion in MI/R. Microvascular dysfunction is one key determinant of $\mathrm{MI} / \mathrm{R}$ injury ${ }^{24-26}$. We used laser Doppler flowmetry to record cardiac microvascular perfusion before and after release of coronary artery ligation. Deletion of $m$ Pges- 1 impaired cardiac perfusion following restoration of coronary blood flow (Fig. 3a, b), without affecting baseline cardiac perfusion. The injured heart tissue of $m$ Pges-1 KO mice showed an increased number of myeloperoxidase (MPO)-positive cells analyzed by immunofluorescent staining (Fig. 3c, d), which was also confirmed by flow-cytometry analysis (Supplementary Fig. 6A, B; the gating strategy for neutrophils was shown in Supplementary Fig. 6C). Heart MPO levels were increased by MI/R (Supplementary Fig. 6D) and mPges-1 deletion further increased MPO levels (Fig. 3e). Enhanced a

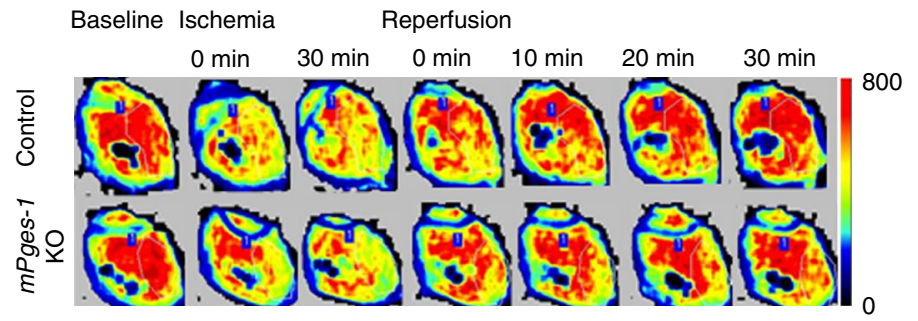

C
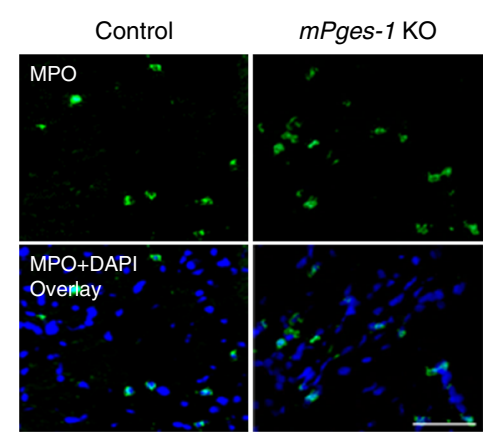

d

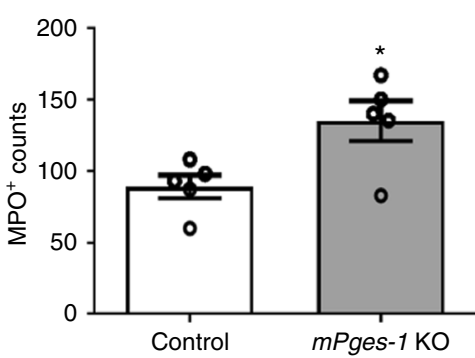

$\mathbf{f}$

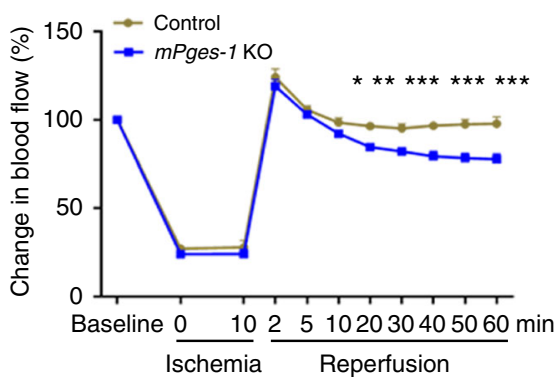

g Baseline Ischemia Reperfusion

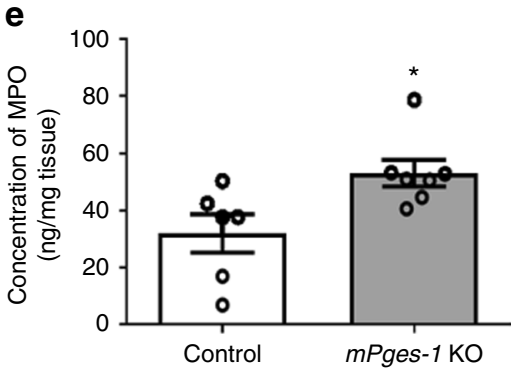

b

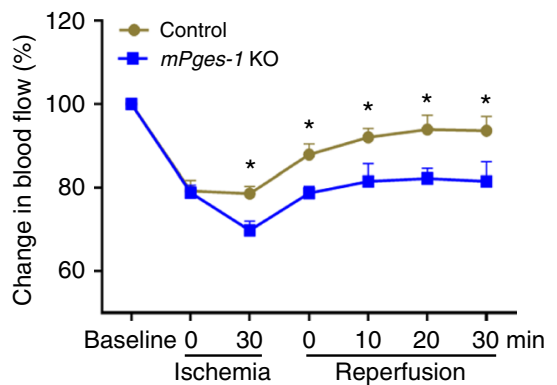

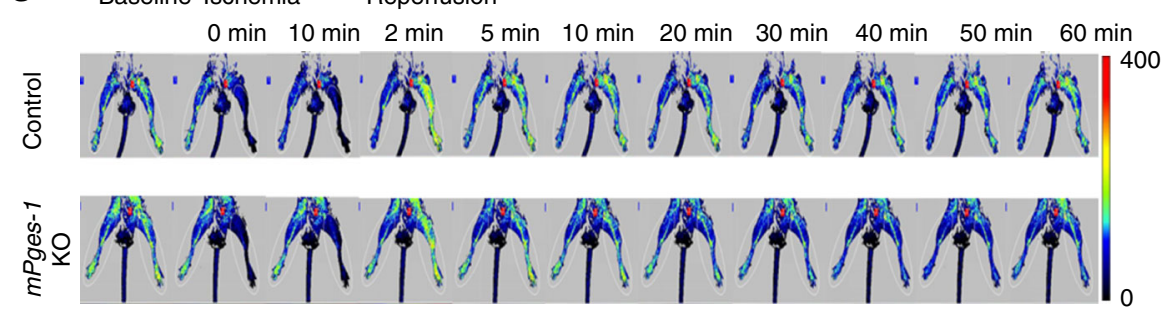

Fig. 3 Essential role of mPGES-1 in preserving microcirculation during I/R. Cardiac microvascular perfusion (percentage change relative to baseline) was recorded at indicated time points before and after release of coronary ligation in mPges- 1 WT and KO mice, as detailed in the Methods. Representative perfusion images at each time point were shown (a). Relative change in blood flow was analyzed (b). Immunofluorescent staining of myeloperoxidase (MPO, in green) was carried out on the heart sections, and representative photos were shown (c). MPO-positive cells (d) and tissue levels of MPO (e) in the ischemic area were quantified. Distal microvascular perfusion was also measured during hindlimb I/R. Microvascular flow relative to baseline was recorded at indicated time points before and after release of the artery ligation (f), with representative perfusion images at each time point shown ( $\mathbf{g}$ ). At the baseline before ischemia, no difference in cardiac perfusion (514 \pm 13 vs $526 \pm 18 \mathrm{PU}, n=17$, 14, unpaired Student's $t$ test) or in hindlimb perfusion ( $86 \pm 6$ vs $90 \pm 4 \mathrm{PU}, n=5$, unpaired Student's $t$ test) was observed between control and mPges- $1 \mathrm{KO}$. Two-way ANOVA with Bonferroni's test (b, $n=12$, $11 ; \mathbf{f}, n=5)$. Unpaired Student's $t$ test $(\mathbf{d}, n=5 ; \mathbf{e}, n=6,7)$. Scale bar is $50 \mu \mathrm{m}$. Error bar indicates SEM 
vascular permeability was also observed in the injured mPges-1 KO hearts (Supplementary Fig. 6E). We utilized another mouse model, in which I/R was induced by ligating the femoral artery and tissue blood flow was monitored in the hindlimb. Deletion of $m$ Pges-1 suppressed the tissue perfusion response relative to control (Fig. 3f, g). Taken together, these results suggest that impaired microcirculatory perfusion may underlie the enhanced MI/R injury in mPges-1 KO mice (Fig. 2). We next examined whether mPGES-1 regulates vasoreactivity and leukocyte-endothelial cell interactions, both of which contribute to microcirculatory function in $\mathrm{I} / \mathrm{R}^{25}$.

mPGES-1 and EP4 mediate arteriolar dilation. Arteriolar size was measured during hindlimb $\mathrm{I} / \mathrm{R}$ in vivo. Reperfusion induced arteriolar dilatation in wild-type mice, however, this response was absent in mPges-1 deficient mice (Fig. 4a). Treatment with GW627368X (an EP4 antagonist), but not antagonists of EP1, EP2 or EP3, inhibited the vascular dilatory response (Fig. 4b). We further characterized isometric vessel tension ex vivo. mPges-1 deletion impaired endothelium-dependent relaxation in response to acetylcholine in resistance arteries, but not in aortas (Supplementary Fig. 7).

mPGES-1/PGE $/$ /EP4 axis limits leukocyte-EC interactions. Mouse lung ECs and peritoneal myeloid cells produced $\mathrm{PGE}_{2}$, mainly derived from mPGES- 1 , in response to IL- $1 \beta$ and complement component $5 \mathrm{a}(\mathrm{C} 5 \mathrm{a})$, respectively (Fig. $5 \mathrm{a}, \mathrm{b})$. The myeloid cells were incubated over a monolayer of ECs and adherent cells were quantified. Treatment of ECs with $10 \mu \mathrm{M}$ Cay10526 (an inhibitor of $\mathrm{PGE}_{2}$ production through the selective modulation of mPGES-1 expression, $\mathrm{IC}_{50}=1.8 \mu \mathrm{M}^{27}$ ) increased cell adhesion, particularly in the presence of C5a (Fig. 5c, d). C5a-stimulated blood leukocyte adhesion was also increased by $m$ Pges-1 deficiency in ECs, as well as in leukocytes (Fig. 5e). Furthermore, I/R of mouse hindlimb induced leukocyte rolling, and such interactions were also increased by mPges-1 deletion (Fig. $5 f$ ). Thus, mPGES-1derived $\mathrm{PGE}_{2}$ limits leukocyte-endothelial cell interactions in the setting of $\mathrm{I} / \mathrm{R}$.

To determine which EP receptor(s) mediate these EC-leukocyte interactions, cells were treated with agonists ${ }^{28,29}$ for $\mathrm{EP} 1 / 3$ (sulprostone, $\mathrm{EC}_{50}=0.42 \mathrm{nM}$ for $\mathrm{EP} 3$, also a weak agonist for $\mathrm{EP} 1$ ), $\mathrm{EP} 2$ (butaprost, $\mathrm{EC}_{50}=32 \mathrm{nM}$ ) or $\mathrm{EP} 4$ $\left(\mathrm{AE} 1-329, \mathrm{EC}_{50}=3.1 \mathrm{nM}\right)$, each at $1 \mu \mathrm{M}$. The EP4 agonist attenuated leukocyte adhesion to ECs from both WT (Fig. 5g) and $m$ Pges- $1 \mathrm{KO}$ mice (Fig. 5h). Similar effects were observed when isolated blood neutrophils were used (Fig. 5i).

Freshly prepared human neutrophils were assessed for adhesion to human microvascular ECs in vitro. Treatment of ECs with Cay10256 increased C5a-induced neutrophil adhesion to the EC monolayer, and activation of EP4 potently inhibited this response, in the absence or presence of Cay10256 (Supplementary Fig. 8).

EC EP4 protects microcirculation and the heart in MI/R. Given that EP4 mediated flow-induced arteriolar dilation (Fig. 4) and leukocyte-EC interactions (Fig. 5), we next examined the role of endothelial EP4 in $\mathrm{MI} / \mathrm{R}$ using mice with postnatal deletion of endothelial Ep4 (EC cKO). Expression of Ep4 mRNA was reduced in ECs isolated from EC cKO mice (Fig. 6a). In hearts after MI/R, EP4 was co-stained with vWF (an EC marker) in control mice, and its expression was reduced in the cKOs (Fig. 6b). Further staining showed low cardiac expression of EP1 and EP2, discrete expression of EP3 and high expression of EP4 in ECs (Supplementary Fig. 9A). Loss of endothelial Ep4 increased infarct size after MI/R (Fig. 6c-e). Levels of ATP (Fig. 6f) in the injured myocardium were reduced in Ep4 cKO mice along with increased levels of ADP and the ratio of ADP to ATP (Supplementary Fig. 6B, C). This coincided with impaired cardiac microvascular perfusion following MI/R (Fig. 6g). More MPO-positive cells were stained in heart sections of the cKO mice (Fig. 6h, i) and cardiac levels of MPO were also increased (Fig. 6j).

We further evaluated the effect of an EP4 agonist (Cay10580) and an EP4 antagonist $(\mathrm{L} 161,982)$ on MI/R injury in Ep4 EC cKO and littermate controls (Supplementary Fig. 10A-C). Treatment with Cay10580 reduced infarct size in both control mice and the cKO mice, however, the cKOs still showed increased injury compared with controls despite the EP4 agonism. EP4 blockade with L161,982 increased infarct size in control mice, to an extent similar to that observed in cKO mice. Similar proportional increase in infarct size was also observed in mice with postnatal global Ep4 deletion (Supplementary Fig. 10D-F). Therefore, EP4 in the endothelium plays a critical, but not exclusive, role in mediating the cardioprotective effect of $\mathrm{PGE}_{2}$ in MI/R.

Misoprostol protects the heart in MI/R. Misoprostol is a PGE analog clinically used to treat NSAIDs (including aspirin)-
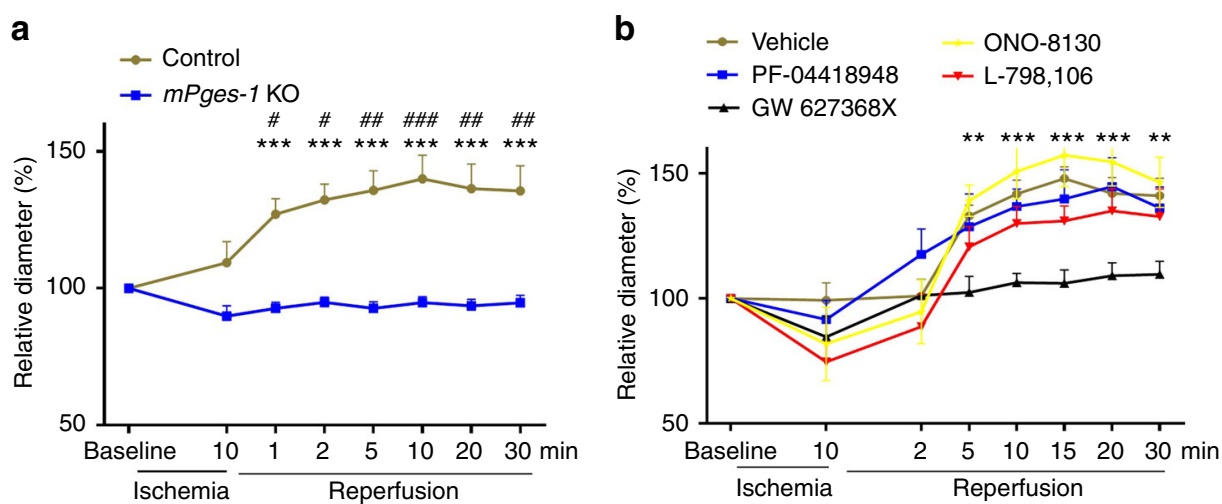

Fig. 4 mPges-1 deletion and PGE receptor antagonism in arteriolar dilation in I/R. Arterioles of 15-25 $\mu \mathrm{m}$ in diameter were randomly selected in the extremity of hindlimb. Vascular response (percentage change of diameter over baseline) to I/R was assessed for mice deficient in $\mathrm{mPges}-1$ (a). The vessel diameter was not different between control $(n=9)$ and mPges-1-1 KO $(n=7)$ mice at baseline $(21.34 \pm 0.72$ vs $20.38 \pm 1.40 \mu \mathrm{m}, p=0.54$, unpaired Student's $t$ test). $\mathbf{b}$ The vascular response was also assessed for WT mice i.v. treated with vehicle $(n=6)$, or an antagonist EP1 (ONO-8130, Ki $=1.9 \mathrm{nM}$, $n=5$ ), EP2 (PF-04418948, IC50 =16 nM, n=5), EP3 (L-798106, Ki=0.3nM, n=7) or EP4 (GW627368X, Ki=100 nM, $n=7$ ), with each drug at $5 \mathrm{mg} / \mathrm{kg}$. There was no difference in baseline vessel diameter between groups. Two-way ANOVA with Bonferroni's test was used. ${ }^{*}$ demarks statistical comparison between control and mPges-1 KO, or between GW 627368X and vehicle. \# shows comparison to baseline. Error bar indicates SEM 
a

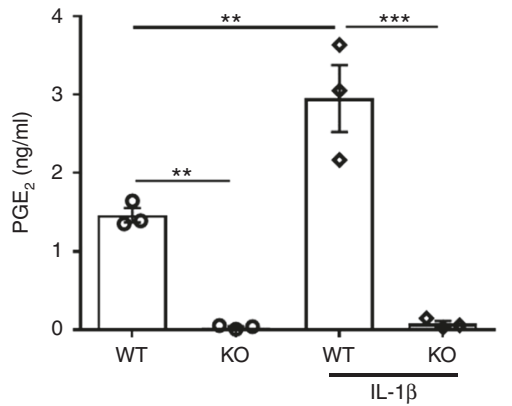

d

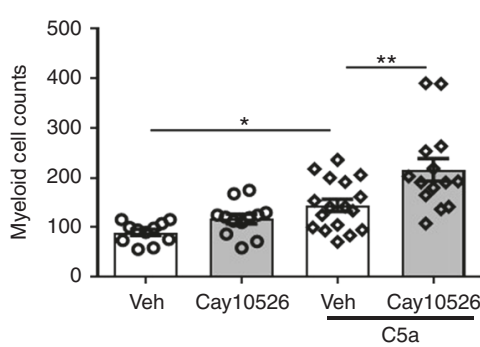

g

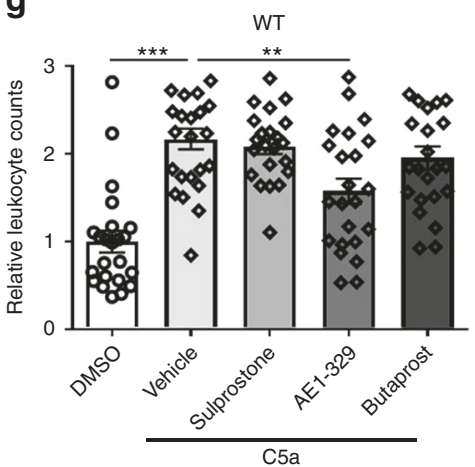

b

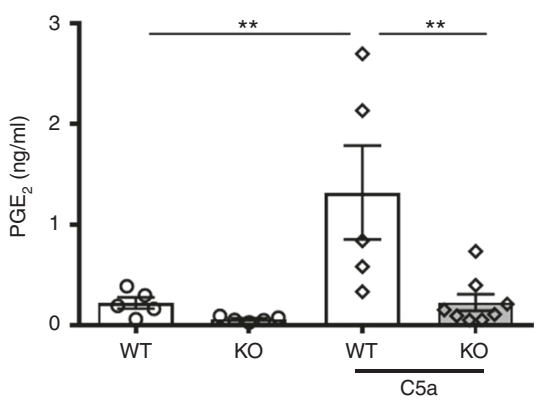

e

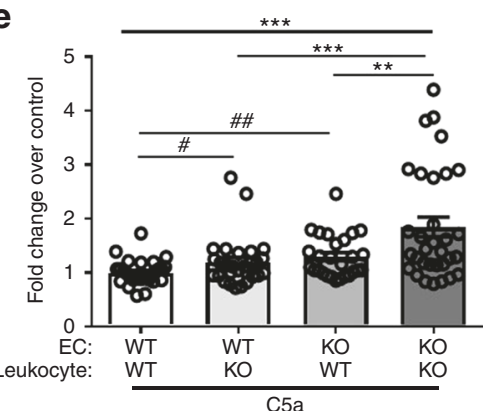

h

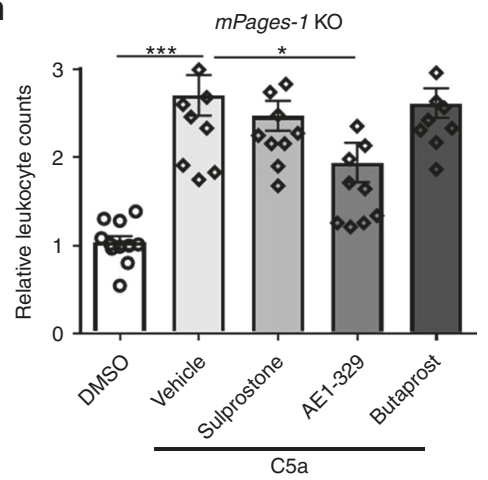

C

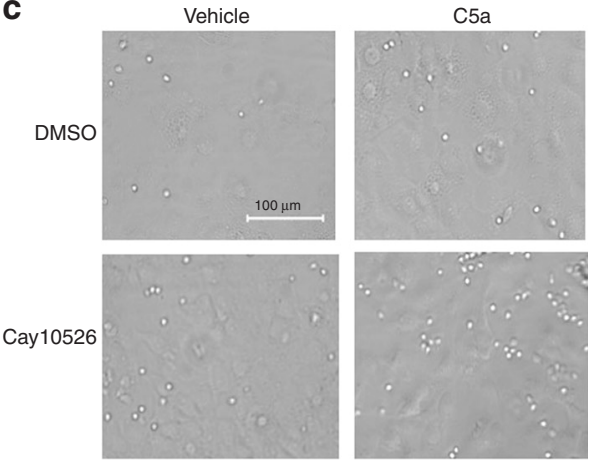

f

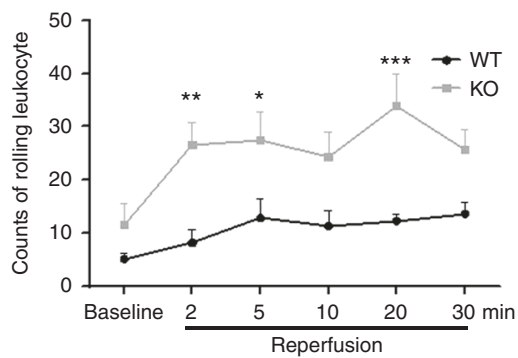

i

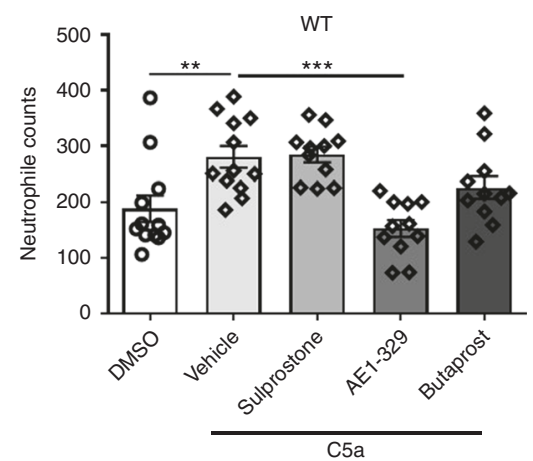

Fig. 5 mPGES-1 derived PGE 2 and EP4 activation limit leukocyte-EC interactions. PGE 2 levels were measured for culture supernatant of mouse ( $m P g e s-1$ WT/KO) lung endothelial cells (ECs) (a) and peritoneal myeloid cells (b). Cells were treated with $10 \mathrm{ng} / \mathrm{ml} \mathrm{IL}-1 \beta$ for $12 \mathrm{~h}$ (a) or with $100 \mathrm{nM} \mathrm{C5a}$ for 30 min (b). Effect of mPGES-1 inhibition on cell adhesion was analyzed. WT ECs were treated with $10 \mu \mathrm{M}$ Cay10526 for $12 \mathrm{~h}$ before peritoneal myeloid cells were applied, and cell adhesion was quantified in absence or presence of $100 \mathrm{nM} \mathrm{C5a}$. Representative photos of cell adhesion for each treatment condition (c) and the quantitative results (d) were shown. Adhesion of blood leukocytes to ECs was determined in vitro in presence of $100 \mathrm{nM}$ C5a (e). The fold change of adherent cells over the number for WT leukocyte to WT EC was presented, with cell genotype labeled below each column.

Leukocyte-endothelial cell interactions were videotaped by intravital microscopy during hindlimb $\mathrm{I} / \mathrm{R}$, and rolling cells were counted prior to and after femoral artery ligation and reperfusion at indicated time points (f), as detailed in the Methods. Effects of agonists for EP1/3 (Sulprostone), EP2 (Butaprost), and EP4 (AE1-329), each at $1 \mu \mathrm{M}$, on in vitro blood leukocyte adhesion to WT (g) or mPges-1 KO (h) EC monolayer were examined in presence of $100 \mathrm{nM}$ C5a. Adhesion of purified blood neutrophils was similarly examined (i). One-way ANOVA with Tukey's multiple comparison tests (a, $n=3 ; \mathbf{e}, n=29,28$, $27,32 ; \mathbf{g}, n=23,24,22,23,23 ; \mathbf{h}, n=11,11,11,11,10 ; \mathbf{i}, n=12,12,11,11,11)$ or Bonferroni's multiple comparison tests $(\mathbf{b}, n=5,5,5,8 ; \mathbf{d}, n=11,12,17,14)$. Two-way ANOVA with Bonferroni's tests $(\mathbf{f}, n=6)$ and unpaired Student's $t$ test $\left(\mathbf{e}, n=29,28,27,32,{ }^{\#} p=0.045\right.$; $\left.{ }^{\#} p=0.0011\right)$. Error bar indicates SEM

induced gastric ulcers. It inhibits formation of gastric lesions in rats $\left(\mathrm{ED}_{50}=0.31 \mu \mathrm{g} / \mathrm{kg}\right)$. When administered following coronary artery ligation, misoprostol $(100 \mu \mathrm{g} / \mathrm{kg})$ reduced infarct size (Fig. $7 \mathrm{a}-\mathrm{c}$ ) and improved cardiac function after $\mathrm{MI} / \mathrm{R}$ in mice (Fig. 7d, e). Flow-cytometry analysis of the injured heart revealed a reduction in infiltrated neutrophils $\left(\mathrm{CD} 11 \mathrm{~b}^{+} \mathrm{Ly}-6 \mathrm{G}^{+}\right.$) (absolute counts: $3430 \pm 502$ vs $1681 \pm 279$; percentage of neutrophils in $\mathrm{CD}_{11 \mathrm{~b}}{ }^{+}$cells: $44.3 \pm 3.1$ vs $33.4 \pm 1.9 \%$ ) after $\mathrm{MI} / \mathrm{R}$ injury (Fig. 7f, g). MPO-positive cells in the heart sections were also reduced by misoprostol treatment (Fig. 7h, i). Misoprostol inhibited leukocyte adhesion in vitro (Fig. 7j) and improved microcirculation following reperfusion (Fig. $7 \mathrm{k}, \mathrm{l}$ and
Supplementary Fig. 11). These observations indicate a key role of $\mathrm{PGE}_{2}$ signaling in preserving microcirculation, while raising the possibility that misoprostol might be repositioned to treat $\mathrm{MI} / \mathrm{R}$ injury.

\section{Discussion}

In this study, we reveal the cardioprotective effect of mPGES-1 in acute MI during cardiac reperfusion. This benefit is attributed to $\mathrm{mPGES}-1$ protection of the microcirculation in $I / R$, which is mediated through the endothelial EP4 receptor. Timely and effective perfusion is critical to salvaging ischemic heart in acute 
a

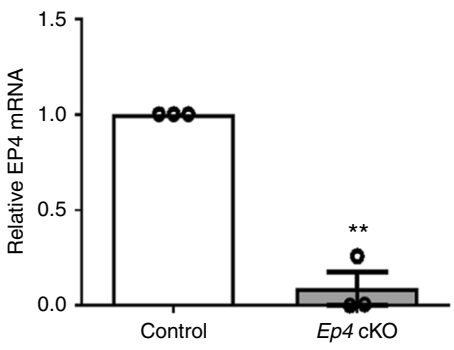

b

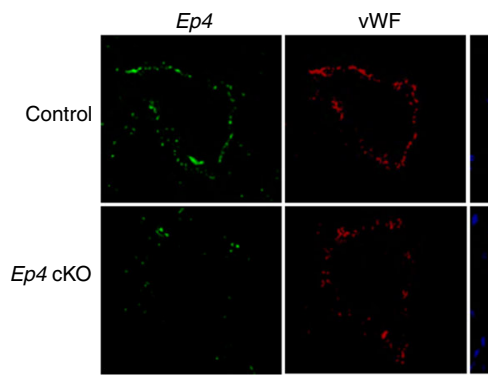

DAPI

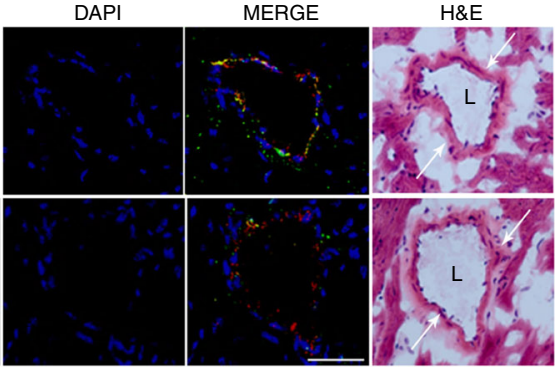

C
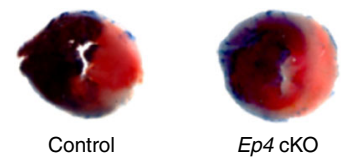

d

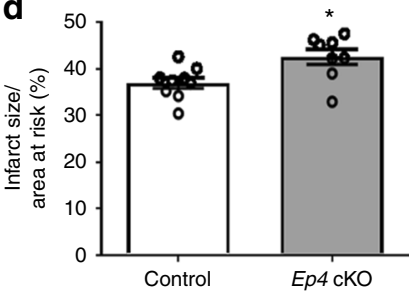

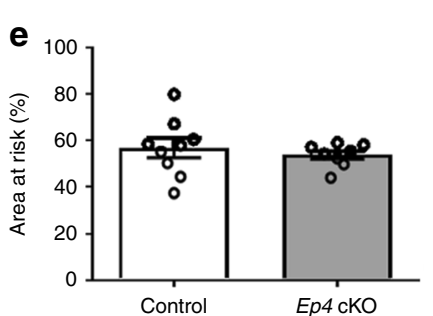

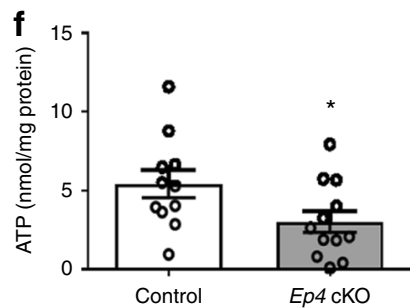

9 Baseline ischemia Reperfusion
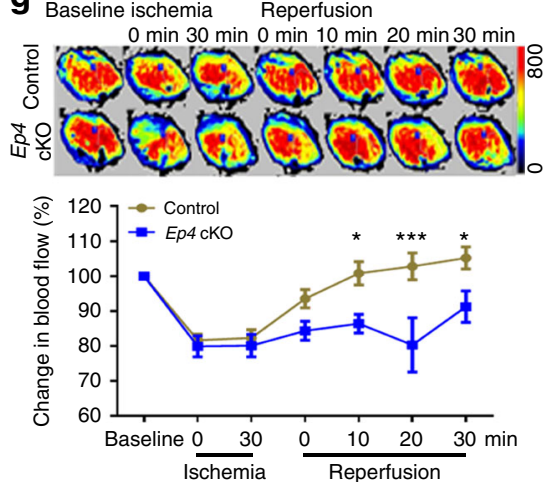

h

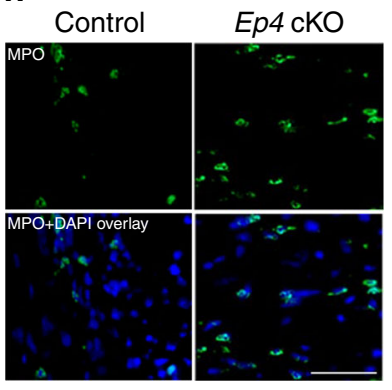

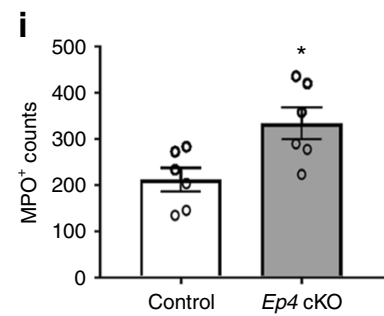

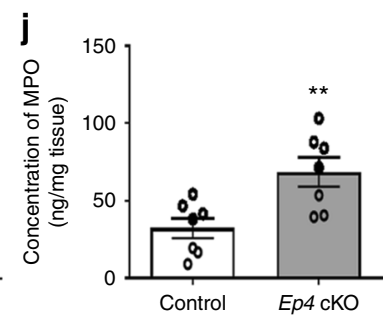

Fig. 6 EC Ep4 deletion impairs microcirculation and increases infarct size in MI/R. Expression of Ep4 was examined by RT-PCR in the mouse lung ECs isolated from tamoxifen treated Ep4 conditional KO mice (cKO) and littermate controls (a). Hearts were isolated $24 \mathrm{~h}$ after $\mathrm{Ml} / \mathrm{R}$, and tissue sections were stained for EP4 (green), VWF (an EC marker, red) and nuclei (DAPI, blue). Representative result from three studies was shown (b). Representative TTC stained sections of the Evans blue perfused hearts were shown (c). Infarct size (d) and AAR (e) were quantified. Levels of ATP in the injured myocardium $24 \mathrm{~h}$ post IR were measured (f). Cardiac blood perfusion during I/R was measured by laser Doppler flow, and representative images were shown above the flow plot ( $\mathbf{g})$. Tissue expression of MPO was stained $(\mathbf{h})$ and the number of MPO-positive cells was analyzed (i). Heart tissue levels of MPO in the ischemic area were quantified (j). Unpaired Student's $t$ test $(\mathbf{a}, n=3 ; \mathbf{d}, \mathbf{e}, n=9,8 ; \mathbf{f}, n=11,12 ; \mathbf{i}, n=6 ; \mathbf{j}, n=7$ ). Two-way ANOVA with Turkey's tests ( $\mathbf{g}, n=15,7)$. Scale bar is $50 \mu \mathrm{m}$. Anatomy of vascular structure is labeled in $\mathbf{b}$ : arrows denote vessel wall, and 'L' denotes lumen. Error bar indicates SEM

MI, and deficiency in microvascular function is associated with clinical events $24,30,31$. Using a standard 30 -min ischemia/24-h reperfusion protocol, this study was focused on acute reperfusion injury, a clinically relevant pathology that might be therapeutically targeted. An extended period after I/R would further increase the clinical relevance of this study. mPGES-1 is required for efficient reperfusion in $\mathrm{MI} / \mathrm{R}$.

The microcirculatory protection of mPGES-1 in I/R may utilize two mechanisms. First, mPGES-1 mediates resistance artery dilatation. COX inhibition-sensitive prostaglandins of unknown identity were reported to mediate arteriolar dilatation ${ }^{32}$. $\mathrm{PGI}_{2}$ is a potent vasodilator ${ }^{28}$ and regulates coronary blood flow in human and mouse ${ }^{25,33} \cdot \mathrm{PGE}_{2}$ was reported either to dilate (EP2, EP4) or constrict (EP1, EP3) large blood vessels depending on vessel types and the receptor subtypes involved ${ }^{34,35}$. Inhibition of mPGES1 in isolated large arteries reduces noradrenaline-induced constriction by increasing $\mathrm{PGI}_{2}{ }^{36}$. Here, we directly measured the diameter of resistance vessels in response to reperfusion. This pathophysiological condition-associated arteriolar dilatation was EP4-dependent, and was abolished by mPges-1 deletion (Fig. 4). A functional study ex vivo provided further evidence that endothelium-dependent dilatation is likely mediated by mPGES-1 in resistance arteries, but not in conduit arteries (Supplementary Fig. 7). Second, mPGES-1-derived $\mathrm{PGE}_{2}$ and EP4 activation limited leukocyte-EC interactions (Fig. 5, Supplementary Fig. 8), adding an additional mechanism for the microcirculatory protection of mPGES-1 in I/R.

The microcirculatory perfusion detected by laser Doppler flow, reflects a combined signal from both ischemic area (due to LAD ligation) and nearby non-ischemic area (flow supplied by other branches of the coronary artery). Interestingly, reduced microcirculatory perfusion was also observed during the cardiac ischemia phase in mPges-1 KOs (Fig. 3b). This likely reflects an mPGES-1 mediated microcirculatory adaption of the nonischemic area to the increased metabolic demand of the ischemic area ${ }^{26}$, which itself indicates a critical role of mPGES-1 in the regulation of microcirculation. However, such pathophysiological compensation in the non-ischemic area might contribute little, if any, to the infarct size. This is because (1) mPGES-1exerts an essential and robust effect in microvascular dilation in the vessels directly affected by I/R (Fig. 4); (2) loss of $m$ Pges-1 triggers a progressive defect in distal limb microcirculation following reperfusion, but without any effect during the ischemia phase (Fig. 3f); and (3) as shown previously, mPges-1 
a
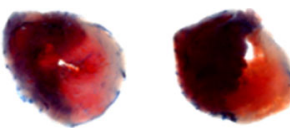

$50 \mu \mathrm{g} / \mathrm{kg}$

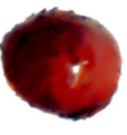

$100 \mu \mathrm{g} / \mathrm{kg}$

b
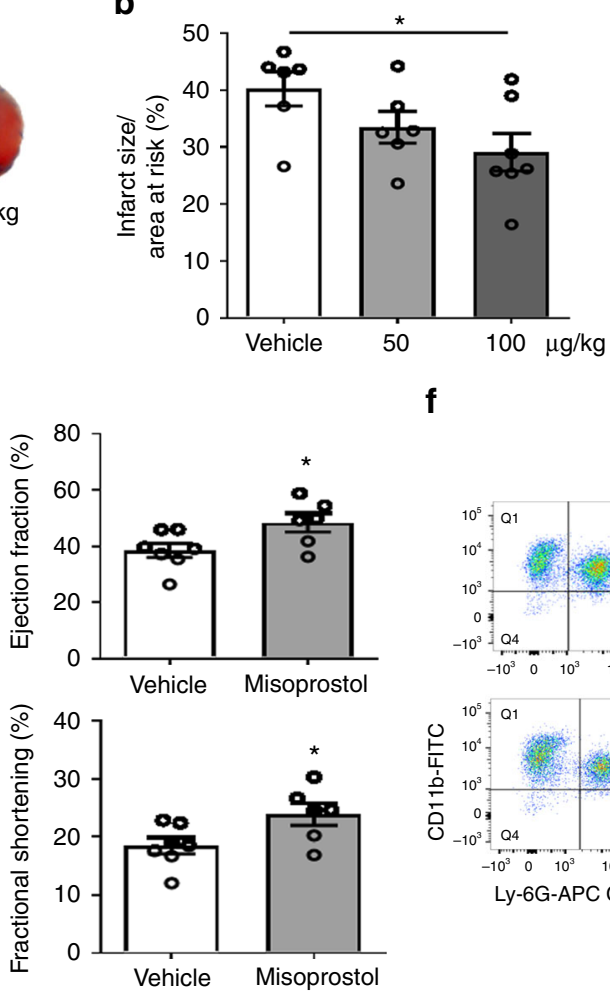

h

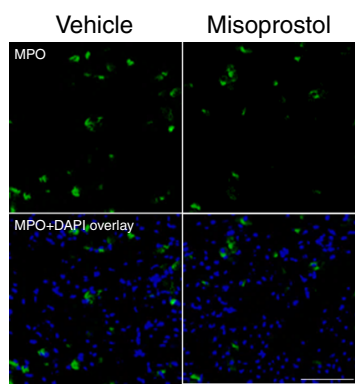

i
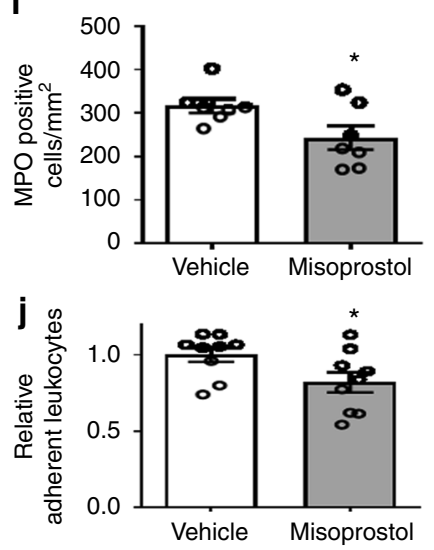

f

C

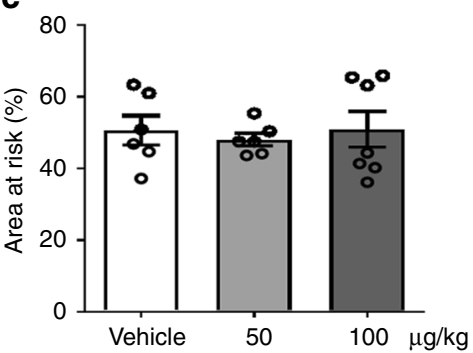

g
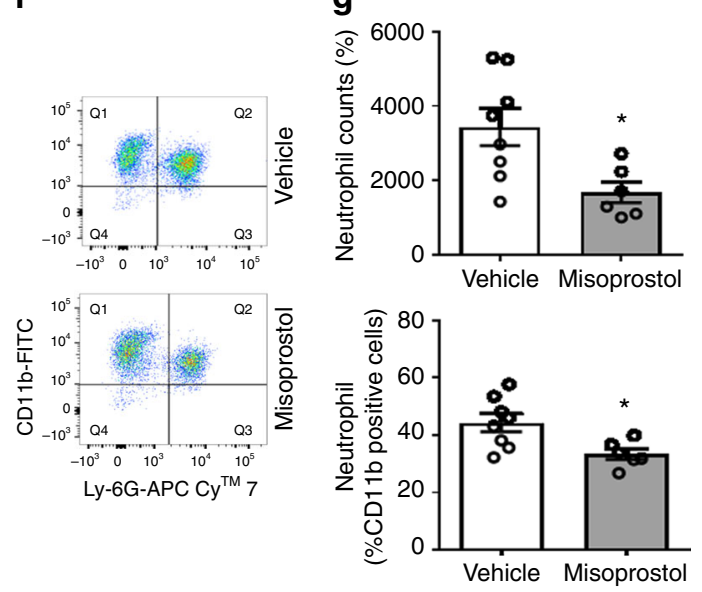

k

Baseline ischemia Reperfusion

$0 \mathrm{~min} 30 \mathrm{~min} 0 \mathrm{~min} 10 \mathrm{~min} 20 \mathrm{~min} 30 \mathrm{~min}$

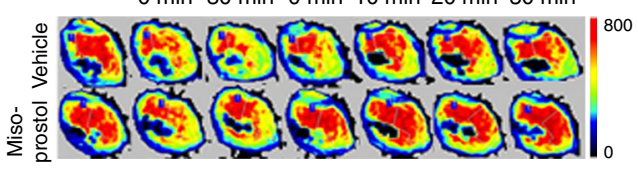

I

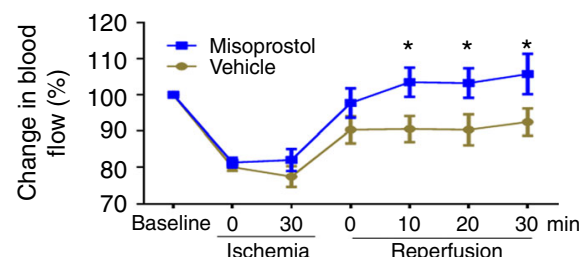

Fig. 7 Misoprostol improves microcirculation and protects the heart in MI/R. WT mice were subjected to MI/R surgery. Vehicle, 50 or $100 \mu \mathrm{gg} / \mathrm{kg}$ misoprostol was injected i.p. following LAD ligation before reperfusion, and additionally at $4,8,12 \mathrm{~h}$ of reperfusion. Representative photos of TTC stained Evans blue perfused hearts were shown (a). Infarct size (b) and AAR (c) were quantified. Ultrasonography was carried out at 24-h post MI/R (d). Ejection fraction (EF) and fractional shortening (FS) were quantified (e). Neutrophils (CD11b + Ly-6G ${ }^{+}$) in the ischemic heart tissue were detected by flow cytometry (f) and quantified as cell counts and percent of neutrophils in $\mathrm{CD}_{11 b^{+}}$cells $(\mathbf{g})$. Immunofluorescent staining of MPO was performed on the heart sections (h) and number of MPO-positive cells was quantified (i). Effect of misoprostol $(10 \mu \mathrm{M})$ on leukocyte adhesion to ECs in vitro (j). In another set of mice, myocardial microcirculatory perfusion was determined during $\mathrm{MI} / \mathrm{R}$, and the representative perfusion images ( $\mathbf{k}$ ) and the statistical graph (I) were shown. One-way ANOVA with Dunn's multiple comparison test (b, $\mathbf{c}, n=6,6,7)$. Unpaired Student's $t$ test $(\mathbf{e}, n=6,7 ; \mathbf{g}, n=6,8 ; \mathbf{i}, n=7 ; \mathbf{j}$, $n=9)$. Two-way ANOVA with Bonferroni's test $(\mathbf{I}, n=16,15)$. Scale bar is $50 \mu \mathrm{m}$. Error bar indicates SEM

deletion does not affect infarct size in mice undergoing acute ischemia only, i.e., without reperfusion ${ }^{19}$. Therefore, preservation of the microcirculation by mPGES-1 during the reperfusion (but not ischemic) phase substantially contributes to the protection against MI/R injury.

The protective role of mPGES-1 in the microcirculation is consistent with an endothelial EP4-dependent mechanism. Here, loss of Ep4 in the endothelium impaired microcirculatory perfusion in MI/R (Fig. 6g). This observation also explains the contrasting impacts on infarct size of global Ep4 $4^{21}$ versus cardiomyocyte-specific Ep4 gene deletion ${ }^{22}$, demonstrating the important contribution from the endothelium. Plasma levels of IL22 were unaltered (Supplementary Fig. 4C), suggesting that the protective effect of mPGES-1 in MI/R does not rely on IL22, a cytokine that restrains sepsis-related systemic inflammation downstream of $\mathrm{PGE}_{2}{ }^{37}$. Our study reveals a protective role of $\mathrm{PGE}_{2}$ in limiting acute sterile inflammation. The mechanism of mPGES1 in protecting against MI/R injury is summarized in Fig. 8.

We found that COX-1, but not COX-2, is most likely to mediate the acute reperfusion injury in vivo (Fig. 1). Previous 


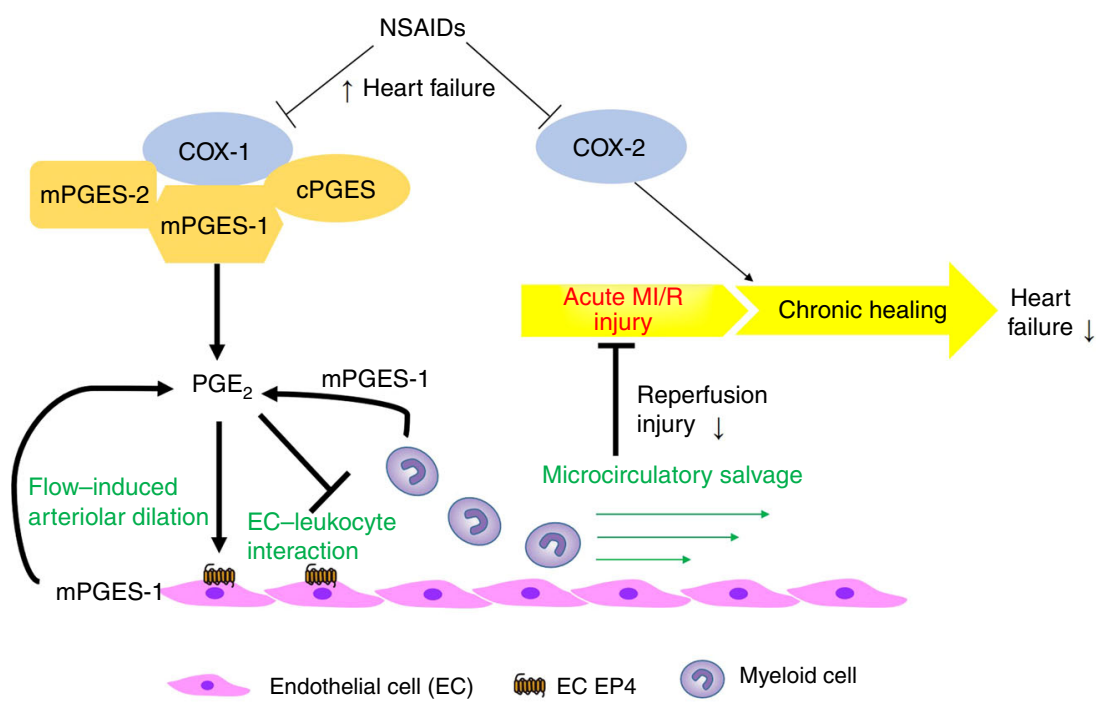

Fig. 8 Schematic illustration of the role of mPGES-1 and EC EP4 in MI/R. Use of NSAIDs is associated with heart failure. In MI/R related heart failure, the COX pathway protects against development of heart failure through acute protection mainly via COX-1/mPGES-1, as shown in the present study (key components/steps highlighted in bold text), and through chronic healing mainly via COX-2, as shown in previous studies. COX-1, the constitutive COX isoform and mPGES-1 contribute substantially to $\mathrm{PGE}_{2}$ biosynthesis and limit acute MI/R injury. mPGES-1, the major source of PGE 2 in endothelial cells and myeloid cells, plays a pivotal role in mediating flow-induced arteriolar dilation and in limiting EC-leukocyte interactions, under I/R. By doing so, mPGES-1 preserves the microcirculation and further constrains MI/R injury. These beneficial effects are mediated in part via endothelial EP4 receptors. $\rightarrow$ denotes 'prostanoid production' or 'positive regulation'; - denotes 'inhibition'; the thick lines highlight the key findings of this study. cPGES: cytosolic PGE synthase

studies have shown that COX-2 mediates a late-phase, protective effect of pre-conditioning on the myocardium ${ }^{15}$ and it is required for chronic cardiac healing after sustained ischemia ${ }^{38}$. Thus, COX-1 may play a critical role in protecting the heart in acute myocardial infarction with reperfusion, whereas COX-2 may contribute to cardioprotection at a later phase of sustained ischemia, consistent with the association of heart failure with NSAIDs, both selective and non-selective for inhibition of COX-2 ${ }^{1}$ (Fig. 8). This mechanism expands our current understanding of the cardiovascular risk of NSAIDs ${ }^{39,40}$.

Our previous studies in mice suggest that chronic inhibition of mPGES-1 is less likely than NSAIDs to cause thrombosis (e.g., myocardial infarction $)^{5}$, and reduces restenosis after vascular injury, due to the increased biosynthesis of $\mathrm{PGI}_{2}{ }^{10,12}$ However, in the setting of myocardial infarction, mPGES-1 inhibition may present a risk to patients undergoing reperfusion. It remains to be determined whether improving microcirculation via EP4 activation will benefit recovery in patients undergoing acute reperfusion.

\footnotetext{
Methods

Mice and drug administration. Mice deficient in Cox-1, Cox-2, or mPges-1, as described $^{5}$, were obtained from the FitzGerald lab (University of Pennsylvania), with permission from original inventors. Cox-1/2 deficient mice were maintained on a mixed C57B $6 \times 129 / \mathrm{Sv}$ genetic background. Knockout $(\mathrm{KO})$ mice and littermate controls were generated from intercrossing heterozygous mice (or with homozygous KO mice). Given that no difference in the characterized phenotypes was detected between the heterozygous and wild-type littermates, these control mice were pooled together for analysis. The $m P g e s-1 \mathrm{KO}$ mice were backcrossed to a C57B6 background for over ten generations. C57B6 wild-type (WT, Ptges ${ }^{+/+}$), or Ptges $^{+l-}$ mice derived from breeding of Ptges ${ }^{+l-}$ with Ptges ${ }^{+l-}\left(\right.$ or $\left.^{-l-}\right)$ were used as controls for the mPges-1 KO mice. Endothelial-specific Ep4 (Ptger4) KO mice were created by crossing Ptger4-floxed mice (Ptger $4 / f)$ with Cdh5-promoter driven CreERT2 (Cdh5 (PAC)-CreERT2+) mice, as described ${ }^{12}$. The resulting endothelial Ep4 conditional-knockout mice (Ptger4f/f Cdh5-CreERT2+: abbreviated, EC Ep4 $\mathrm{cKO}$, or cKO) and littermate controls (Ptger4f/f Cdh5-CreERT2-, Ctl) were postnatally treated with tamoxifen to induce endothelial Ep4 deletion. Inducible global Ep4 KO mice were similarly prepared with used of R26CreER mutant mice from the Jackson lab, which have a tamoxifen-inducible CreER driven by the endogenous mouse Gt(ROSA)26Sor promoter. In all cases, transgenic mice deficient in the indicated gene were compared with age-, and sex-matched control mice.
}

Celecoxib $(100 \mathrm{mg} / \mathrm{kg})$ or vehicle ( $1 \%$ methyl cellulose) was orally gavaged before $\mathrm{MI} / \mathrm{R}$ surgery and again $20 \mathrm{~h}$ after MI/R. For the misoprostol study, male C57B6 mice (8-10 weeks old) were subjected to left coronary artery ligation and randomized to receive 50 or $100 \mu \mathrm{g} / \mathrm{kg}$ misoprostol or vehicle (saline) via i.p. injection after ligation and at $4,8,12 \mathrm{~h}$ after reperfusion. Mice were euthanized $24 \mathrm{~h}$ after reperfusion for sample collection.

For assessment of EP4 antagonism, 8-week-old mice were subjected to MI/R surgery and treated by intraperitoneal injection of CAY10580 (a selective EP4 agonist, Cayman Chemical, Ann Arbor, USA; $200 \mu \mathrm{g} / \mathrm{kg}$ body weight, twice a day), L-161,982 (an EP4 selective antagonist, Sigma-Aldrich, USA; $5 \mathrm{mg} / \mathrm{kg}$ body weight, once a day), or vehicle ( $2 \%$ ethanol/DMSO in PBS) immediately after ligation.

All animal protocols complied with all relevant ethical regulations and were approved by the Institutional Animal Care and Use Committee, the Experimental Animal Center, Fuwai Hospital, National Center for Cardiovascular Diseases, China. The experimental performers were blind to genotype/treatment grouping information during the experimental conduction and quantification.

Myocardial ischemia/reperfusion model. Induction of myocardial I/R injury was conducted using a method without artificial ventilation ${ }^{41}$. Briefly, mice were anesthetized with $3 \%$ isoflurane inhalation followed by $1.5-2 \%$ isoflurane inhalation for anesthesia maintenance. The mice were placed in a supine position. The skin on the left thorax was slit, and the thoracic muscle was simply separated. Then, the thoracic cage was promptly exposed via thoracotomy through the 4 th intercostal space on the left. After the pericardium was opened, the mouse heart was exposed, and the left anterior descending (LAD) coronary artery was ligated with a slipknot at $\sim 3 \mathrm{~mm}$ from the origin using a 7-0 silk suture. The success of the ligation was confirmed by the anterior wall of the LV turning pale coincident with ST-segment elevation on the electrocardiogram (ECG). The heart was then quickly placed back into the thoracic space, followed by manual evacuation of air and the chest closed with a $4-0$ suture. The internal end of slipknot suture was cut as shor as possible, and the other end of the suture was $\sim 0.8 \mathrm{~cm}$ long and remained outside of the chest. Anesthesia was then stopped, and the animals were allowed to recover. After $30 \mathrm{~min}$ of ischemia, mice were re-anesthetized, the slipknot was released by pulling the long end of the suture smoothly and gently until a feeling of release was sensed, at which time, the myocardial reperfusion began. The suture was removed to avoid prolonged tissue injury. At $24 \mathrm{~h}$ post I/R, cardiac function and the ventricular structure were determined via echocardiography (VisualSonics VeVo 2100 Imaging System) by evaluating the ejection fraction (EF), left ventricular fractional shortening (FS), and left ventricular anterior wall thickness [LVAW; s(systolic)/d (distolic)]. Mortality was similar across the groups, at $\sim 20 \%$. At $24 \mathrm{~h}$ post I/R, the LAD was reoccluded in the previous position, and 2\% Evans blue dye (Sigma, Darmstadt, Germany) was injected into the heart cavity through the ascending aorta. The mouse was then euthanized, and its heart was harvested and rinsed in PBS. The heart was then frozen at $-80^{\circ} \mathrm{C}$ for $30 \mathrm{~min}$ and cut transversely into six slices below the ligature. The slices were incubated with $1 \%$ 2,3,5-triphenyltetrazolium chloride (TTC, Amresco, USA) at $37^{\circ} \mathrm{C}$ for $10 \mathrm{~min}$ in the dark room and 
then fixed with $10 \%$ formalin for $2 \mathrm{~h}$. A stereomicroscope (Zeiss, Germany) was used to take pictures. The areas of the ischemic region, infarcted tissue, and LV were measured and calculated using the Image-Pro Plus 6.0 software (Media Cybernetics).

Detection of microcirculatory perfusion. To determine microcirculatory blood flow during myocardial (M) I/R, mice were anesthetized using pentobarbital sodium, intubated and ventilated with a positive-pressure respirator. After removing the thoracic muscle, the thoracic cage was opened via thoracotomy through the 4th intercostal space on both sides and the midsternum. The heart was then completely exposed by cutting off 2-4 ribs on both sides and removing the anterior thorax wall. To induce heart ischemia, the LAD coronary artery was ligated with a $7-0$ silk suture $2-3 \mathrm{~mm}$ from the origin. Thirty minutes later, the ligature was removed to allow reperfusion of the ischemic myocardium. To monitor microcirculatory perfusion, hearts were scanned using laser Doppler flowmetry (PeriCam PSI System, Perimed, Sweden) at baseline (prior to ligation), 0 and 30 min post ligation, and $0,10,20$, and 30 min post reperfusion. Left ventricular epicardial blood flow at the ischemic part was determined and analyzed blindly.

To measure microcirculatory response to hindlimb $\mathrm{I} / \mathrm{R}$, mice were anesthetized by pentobarbital sodium, and the left femoral artery was exposed with blunt dissection. Hindlimb ischemia (HLI) was induced via ligating the artery at the proximal site of the femoral artery bifurcation, followed by reperfusion via releasing the suture $10 \mathrm{~min}$ later. Blood flow in the distal extremity of hindlimb during the I/R was determined by PeriCam PSI System before and immediately after the ligation and at different time points after reperfusion. For misoprostol study, mice were injected i.p. with misoprostol $(100 \mu \mathrm{g} / \mathrm{kg}$, in saline) $10 \mathrm{~min}$ before ischemia.

Measurement of vascular response in hindlimb I/R. Mice (18-20 weeks) were anesthetized by i.p. injection of pentobarbital sodium $(80 \mathrm{mg} / \mathrm{kg})$. The mice were fixed in a supine position. Then the arterioles downstream of the femoral artery were gently exposed. Mice were injected i.v. via angular vein with Rhodamine $6 \mathrm{G}$ (Sigma, Darmstadt, Germany; $5 \mathrm{mg} / \mathrm{kg}$ in saline) or with Rhodamine $6 \mathrm{G}$ plus an antagonist of EP1 (ONO-8130 ${ }^{42}, \mathrm{Ki}=1.9 \mathrm{nM}, \mathrm{MW}=500.6$, Cayman, USA), EP2 $\left(\mathrm{PF}-04418948^{43}, \mathrm{IC} 50=16 \mathrm{nM}, \mathrm{MW}=409.4\right.$, Selleck, USA), EP3 (L-798106 ${ }^{44}$, $\mathrm{Ki}=0.3 \mathrm{nM}, \mathrm{MW}=536.4$, Cayman, USA), or EP4 $\left(\mathrm{GW} 627368 \mathrm{X}^{45}, \mathrm{Ki}=100 \mathrm{nM}\right.$, $\mathrm{MW}=544.6$, MedChem Express, USA), all at $2.5 \mathrm{mg} / \mathrm{kg}$ (in saline with $10 \%$ DMSO, V/V), $\sim 2$ min before imaging. One arteriole with diameter between 15 and $25 \mu \mathrm{m}$ was randomly chosen for measuring vascular reactivity under a Nikon microscope (E400, Japan). The mouse position was then fixed, and hindlimb ischemia was induced by applying a rubber band tie around the ipsilateral thigh, and ischemia was verified by complete blockade of blood flow verified with the microscope (excited with green fluorescence). Ten minutes later, reperfusion was induced by release of the rubber band. The microvessel response was videotaped with a JPLY camera at a magnification of $200 \times$. Customized image analysis software was used to assess the vessel diameter. The vessel diameter was measured prior to ligation, $10 \mathrm{~min}$ post ligation, and, 1 (for some experiments), 2, 5, 10, 15 (for some experiments), 20 and $30 \mathrm{~min}$ post reperfusion.

Isometric vessel tension study. After mice were sacrificed by overdosing of pentobarbital sodium $(100 \mathrm{mg} / \mathrm{Kg})$, aortas and the second order branches of mesenteric arteries were isolated for vessel tension studies ${ }^{46}$. Briefly, the arteries were cut into vessel rings, with each ring $\sim 2 \mathrm{~mm}$ in length, in ice-cold KrebsRinger bicarbonate solution $\left(118.3 \mathrm{mM} \mathrm{NaCl}, 4.7 \mathrm{mM} \mathrm{KCl}, 2.5 \mathrm{mM} \mathrm{CaCl}_{2}, 1.2 \mathrm{mM}\right.$ $\mathrm{MgSO}_{4}, 1.2 \mathrm{mM} \mathrm{KH}_{2} \mathrm{PO}_{4}, 25 \mathrm{mM} \mathrm{NaHCO}$, and $11.1 \mathrm{mM}$ glucose). The rings were suspended in the chambers of a multi myograph (620 M, Danish, Myo Technology A/S, Aarhus, Denmark) using two tungsten wires. Each chamber contained $5 \mathrm{~mL}$ Krebs solution which was maintained at $37^{\circ} \mathrm{C}$ and constantly bubbled with $95 \% \mathrm{O}_{2}$ and $5 \% \mathrm{CO}_{2}$. At the beginning of the experiments, each vessel ring was brought to its optimal tension ( $\sim 2.5 \mathrm{mN}$ for aortas, $\sim 1 \mathrm{mN}$ for mesenteric arteries). After $60 \mathrm{~min}$ equilibration, the arteries were firstly contracted with $60 \mathrm{mM} \mathrm{KCl}$, followed by three washes with warmed Krebs solution. To determine the endotheliumdependent vasodilation, the vessels were contracted with $3 \mu \mathrm{M}$ phenylephrine (PE), followed by adding acetylcholine (ACh) at different concentrations $(0.003-10 \mu \mathrm{M})$. The vessels were then washed three times again, and L-NAME $(100 \mu \mathrm{M})$ and indomethacin $(10 \mu \mathrm{M})$ were added to inhibit endothelial cell activities. After 30min incubation with L-NAME and indomethacin, the endothelium-independent vasodilation was examined by determining vasodilation of sodium nitroprussiate (SNP, a NO donor) to U46619 (100 nM) or PE $(3 \mu \mathrm{M})$.

Intravital microscopy of leukocyte adhesion. Leukocyte adhesion was monitored in vivo by intravital microscopy of the femoral artery. Mice were injected i.v. via angular vein with Rhodamine 6G (Sigma, Darmstadt, Germany; $5 \mathrm{mg} / \mathrm{kg}$ in saline) before femoral artery ligation. Leukocyte adhesion was live-imaged and recorded with a Nikon microscope (E400; excited with green fluorescence) that was connected to a Nikon camera (S1TC01M). Time-lapse video was analyzed offline by a technician blind to mouse grouping information, the number of rolling leukocytes was counted for $1 \mathrm{~min}$ at different time points (prior to ligation; 2, 5, 10, 20, and 30 min post reperfusion).

Bone marrow transplantation. Briefly, recipient mice (WT or mPges-1 KO) were irradiated with one sub-lethal dose of $10 \mathrm{~Gy}$ (Gammacell $40{ }^{137} \mathrm{Cs} \gamma$-irradiation source) for a period of 10-12 min. Each recipient was reconstituted via retro-orbital vein injection of $5 \times 10^{6} \mathrm{WT}$ or KO bone marrow cells. Mice were then allowed to recover for $\sim 5$ weeks, including 1-2 weeks on antibiotic treatment $(1-2 \mathrm{mg} / \mathrm{ml}$ gentamycin or neomycin $1.1 \mathrm{mg} / \mathrm{ml}$ and polymyxin B sulfate $1000 \mathrm{U} / \mathrm{ml}$ in drinking water). The bone marrow chimeric mice were then subjected to MI/R injury study.

Analysis of cardiac neutrophils by flow cytometry. Hearts were isolated $24 \mathrm{~h}$ post MI/R, and about $20 \mathrm{mg}$ of heart tissue was cut from the apex. Minced tissue was then digested with collagenase $(0.895 \mathrm{mg} / \mathrm{ml}$, Type II, Sigma, USA) and protease $\left(0.5 \mathrm{mg} / \mathrm{ml}\right.$, Type XIV, Sigma, USA) in a $37^{\circ} \mathrm{C}$ shaker for $7 \mathrm{~min}$. The digestion mix was filtered using a $74-\mu \mathrm{m}$ strainer and centrifuged at $200 \times g$ for $5 \mathrm{~min}$ at $4^{\circ} \mathrm{C}$. The cell pellet was resuspended by $100 \mu \mathrm{l}$ FACS buffer, $50 \mu \mathrm{l}$ was stained with an antibody mixture (PE Rat Anti-Mouse CD45,1:50, 553081; FITC Rat Anti-Mouse CD11b, 1:50, 553310; APC-Cy ${ }^{\mathrm{TM}} 7$ Rat Anti-Mouse Ly-6G,1:50, 560600; BD Biosciences; New York, USA) for 30 min on ice in dark. Then each sample was added with $250 \mu \mathrm{l}$ FACS buffer, filtered with a $74 \mu \mathrm{m}$ nylon membrane and analyzed by flow cytometer at a constant flow rate and a fixed collection time.

Cell study. Myeloid cell isolation: C57B6 mice were injected intra-peritoneally with 4\% Brewer modified Thioglycollate medium (BD, Franklin Lakes, NJ, USA) (1 mL/mouse). After $5 \mathrm{~h}$, peritoneal ascites was washed out with PBS containing 0.1\% BSA for collection, followed by centrifugation $(200 \times g, 3 \mathrm{~min})$. The sedimentary leukocytes, which were mainly myeloid cells, were harvested and used for cell adhesion study.

Blood leukocyte isolation: Human or mouse whole blood were lysed in red blood cell lysis buffer (BD, Franklin Lakes, NJ, USA) for $5 \mathrm{~min}$ at room temperature, followed by centrifugation $(1000 \times g, 2 \mathrm{~min})$. Cell pellets (leukocytes) were resuspended in saline for experiments.

Mouse neutrophil isolation: Mice were anesthetized by intraperitoneal injection of pentobarbital sodium $(80 \mathrm{mg} / \mathrm{kg}$ body weight). Blood was collected by retroorbital bleeding; $1 \mathrm{ml}$ of blood was mixed with $7 \mathrm{ml}$ HBSS (Corning, New York, USA) containing $15 \mathrm{mM}$ EDTA, followed by $500 \times g$ centrifugation for $10 \mathrm{~min}$, at room temperature. The cell pellet was lysed by mixing with $5 \mathrm{ml} \mathrm{BD}$ red blood lysis buffer at RT for $5 \mathrm{~min}$, followed by adding $5 \mathrm{ml} \mathrm{RPMI} \mathrm{(Gibco,} \mathrm{Waltham,} \mathrm{MA,} \mathrm{USA)}$ media containing $2 \%$ FBS. After 10 -min centrifugation at $200 \times g$, the cell pellet was washed once with isolation buffer (HBSS with $2 \mathrm{mM}$ EDTA and 1\% BSA) and then centrifuged at $400 \times g$ for $5 \mathrm{~min}$, at room temperature. The cell pellet was gently resuspended in $6 \mathrm{ml} \mathrm{45 \%}$ Percoll (GE, New York, USA), loaded onto Percoll gradient $(81-62-45 \%)$, and centrifuged at $1500 \times g$ for 30 min with break off. The neutrophil layer was collected to $10 \mathrm{ml}$ isolation buffer. After $10 \mathrm{~min}$ centrifugation at $1200 \times g$, cells were resuspended in $5 \mathrm{ml}$ RPMI (containing $10 \% \mathrm{FBS}$ ).

Human neutrophil isolation: Human neutrophils were freshly prepared at room temperature from healthy donors after obtaining informed consent. Neutrophils were isolated using Lymphocyte-poly isolation media (Cedarlane Labs, Ontario, Canada) from EDTA-anticoagulated peripheral blood. Briefly, blood was loaded to the separation media at a ratio of 1:1 in volume in a conical tube, and was centrifuged at $500 \times g$ for $35 \mathrm{~min}$ with the break off. The neutrophil layer was collected into $40 \mathrm{ml}$ of PBS without $\mathrm{Ca}^{2+}$ and $\mathrm{Mg}^{2+}$, and centrifuged at $400 \times g$ for $10 \mathrm{~min}$. The pellet was resuspended in $1 \mathrm{ml}$ PBS, subjected to red blood cell lysis buffer, and was then centrifuged at $200 \times g$ for $10 \mathrm{~min}$. Cell pellet was washed in PBS and resuspended in PBS.

Cell adhesion study: Endothelial cells were seeded and cultured in a 96-well flat bottom plate to reach full confluence. The cells were treated with indicated drugs (dissolved in DMSO) in a medium containing 5\% FBS for $2 \mathrm{~h}$, except for mPGES-1 inhibitor (Cay10526), which was incubated for $12 \mathrm{~h}$. Then, complement factor 5a (C5a) in PBS was added to a final concentration of $100 \mathrm{nM}$, and incubated for 30 min. Peritoneal or blood leukocytes, prepared in RPMI medium containing $10 \%$ FBS, were then loaded to the EC monolayer culture, and incubated for another $30 \mathrm{~min}$. The coculture was washed three times in RPMI. The adherent leukocytes were then counted in a field of view at the center of each well under Leica microscope (dmi4000B; excited with green fluorescence) at $\times 100$ magnification.

The studies using human whole blood and cells complied with all relevant ethical regulations. Ethical approval was obtained from the Institutional Review Board, Fuwai Hospital, National Center for Cardiovascular Diseases, China. Informed consent was obtained from all human participants.

Immunofluorescence staining. Hearts were collected, frozen, embedded and sectioned at a thickness of $10 \mu \mathrm{m}$. For each sample, the sections were serially gathered at $200 \mu \mathrm{m}$ intervals below the ligature for a total of four levels. Tissue sections were fixed with $95 \%$ ethanol for $15 \mathrm{~min}$, and then incubated at room temperature for $90 \mathrm{~min}$, with a goat serum containing $0.3 \%$ Triton X-100 for blocking and membrane rupture. The heart sections were incubated with rabbit anti-myeloperoxidase (MPO) antibody (1:50; Abcam, ab9535, Cambridge, UK) at $4{ }^{\circ} \mathrm{C}$ overnight, rinsed with PBST, and then incubated with Alexa Fluor 488 goat 
anti-rabbit antibody (1:200; ZSGB-BIO, ZF-0511, Beijing, China) for $1 \mathrm{~h}$ at $37^{\circ} \mathrm{C}$. For dual staining, the heart sections were incubated at $4{ }^{\circ} \mathrm{C}$ overnight in a mixture of sheep anti-Von Willebrand factor (VWF) antibody (1:100; Abcam, ab11713, Cambridge, UK, for endothelial cell delineation) and rabbit antibody against EP1 (101740), EP2 (101750), EP3 (101760), or EP4 (101775). All EP1-4 antibodies were from Cayman (Michigan, USA) and used at a dilution of 1:200. The secondary antibodies used were FITC 488 goat anti-rabbit antibody and Alexa Fluor 594 donkey anti-sheep IgG (1:200; Invitrogen, A-11016, California, USA). Coverslips were mounted with a VectaShield medium containing DAPI to stain nuclei. The sections were imaged using a Zeiss inverted fluorescence microscope (AXI0; Zeiss, Germany) equipped with a Zen software. MPO-positive cells were counted for 4 levels of each sample using the Image-Pro Plus 6.0 software (Media Cybernetics, Inc., Rockville, MD, USA).

ATP and ADP measurement. Heart tissue samples at area of risk or that from matching part of naive animals were harvested. ADP and ATP levels were measured using colorimetric/fluorometric assay kit for ATP (MAK190, SIGMA, CA, USA) and ADP (MAK081, SIGMA, CA, USA), according to the manufacturer's instructions. Protein concentration of cell lysate was determined using BCA protein assay. ATP abundance was normalized to tissue weight.

Analysis of MPO concentrations. Heart tissue samples at area of risk or that from matching part of naive animals were harvested. About $10 \mathrm{mg}$ tissue of each sample was lysed by adding $100 \mu \mathrm{l}$ lysis buffer $(200 \mathrm{mM} \mathrm{NaCl}, 5 \mathrm{mM}$ EDTA, $10 \mathrm{mM}$ Tris, $10 \%$ glycerin, $1 \mathrm{mM}$ PMSF, $1 \mu \mathrm{g} / \mathrm{ml}$ leupeptin and $28 \mu \mathrm{g} / \mathrm{ml}$ aprotinin, PH 7.4). After homogenization, sample was centrifuged twice $\left(1500 \times g, 4^{\circ} \mathrm{C}, 15 \mathrm{~min}\right)$ to avoid contamination of cell debris. The level of MPO was then determined spectrophotometrically by commercial MPO ELISA Kit (HK210, Hycult Biotechnology, Uden, Netherlands) according to the manufacturer's instruction. MPO concentration was normalized to tissue weight.

RT-PCR. Cellular RNA was extracted by TRIzol (Invitrogen, California, USA) according to the manufacturer's instruction. PrimeScript ${ }^{\text {tu }}$ RT Master Mix (TaKaRa, Tokyo, Japan) was used to prepare cDNA. Quantitative RT-PCR was performed using TransStart Tip Green qPCR SuperMix (TransGen, Beijing, China). The sequences of the primers used were as follows: $\beta$-actin, ACCTTCTACAATGAGCTGCG (forward) and CTGGATGGCTACGTACATGG (reverse); Ep4, GCCATCAGGATTGCTTCT (forward) and ACCAACAGGAC ACTCTCA (reverse); Cox-1, CACTCGCCTCATCCTTATAG (forward), GTTCCTACCTCCACCAATC (reverse); Cox-2, CСTTCTCCAACCTCTCCTA (forward), ACACCTCTCCACCAATGA (reverse).

Prostanoid determination. $\mathrm{PGE}_{2}$, was determined by liquid (L) chromatography (C)-tandem mass $(\mathrm{M})$ spectrometry $(\mathrm{S})^{47}$. UPLC BEHC18 column $(1.7 \mu \mathrm{m}, 100 \times$ $2.1 \mathrm{~mm}$ i.d.) consisting of ethylene-bridged hybrid particles (Waters, Milford, MA) was used for Chromatographic separations. The isolates were analyzed using a 5500 QTRAP hybrid triple quadrupole linear ion trap mass spectrometer (AB Sciex, Foster City, CA) equipped with a turbo ion spray electrospray ionization source. EDTA-anticoagulated plasma samples were harvested $24 \mathrm{~h}$ post MI/R surgery and used for $\mathrm{PGE}_{2}$ determination. Serum-free cell culture media was used to culture primarily isolated endothelial cells or neutrophils, for indicated duration of time. After 5 min centrifugation at $6000 \times g$, supernatant was stored at $-80^{\circ} \mathrm{C}$ for $\mathrm{PGE}_{2}$ determination.

Twenty-four hours urine was collected from mice subjected to MI/R injury. Biosynthesis of $\mathrm{PGE}_{2}$ and $\mathrm{PGI}_{2}$ was estimated via measuring their urinary metabolites, tetranor-PGEM and 2,3-dinor-6-keto-PGF ${ }_{1 \alpha}$, respectively, by liquid chromatography-tandem mass spectrometry ${ }^{48}$.

IL-22 measurement. Plasma IL-22 was measured with mouse IL-22 Quantikine ELISA Kit (R\&D Systems, Minnesota, USA), following the manufacturer's instruction.

Modified miles assay. Briefly, $m$ Pges $-1 \mathrm{WT}$ and KO mice were subjected to coronary artery ligation for $30 \mathrm{~min}$, and $1 \%$ Evans blue $(200 \mu \mathrm{L})$ was injected intravenously by retro-orbital injection before reperfusion ${ }^{49}$. Mice were euthanized $4 \mathrm{~h}$ after reperfusion and perfused through the aorta with citrate buffer $(\mathrm{pH} 4)$. The area at risk of left ventricular (below the ligation) including ventricular septum was dissected, and Evans blue was extracted in $1 \mathrm{~mL}$ of formamide for $18 \mathrm{~h}$ at $70^{\circ} \mathrm{C}$. After centrifugation, absorbance was measured at $620 \mathrm{~nm}$ by using a spectrophotometer. The amount of extravasated Evans blue $(\mu \mathrm{g})$ was determined from a standard curve and normalized to heart tissue weight (g).

Statistical analysis. Statistical analysis was performed using GraphPad Prism 5 software (GraphPad Software Inc., San Diego, California, USA). Student's $t$-test (two-tailed, unpaired) was used for two-group comparisons. When a time factor was involved, two-way ANOVA was used for data comparisons. Multiple-group comparisons were made using one-way ANOVA. Following either one-way or twoway ANOVA, post hoc tests were performed with Bonferroni correction unless otherwise indicated in figure legends. Data were expressed as mean \pm standard error of mean (SEM). Differences were considered statistically significant at $P<$ 0.05. Statistical significance is demarked as ${ }^{\star} P<0.05,{ }^{* *} P<0.01$, ${ }^{* *} P<0.001$.

Reporting Summary. Further information on experimental design is available in the Nature Research Reporting Summary linked to this article.

\section{Data availability}

The data that support the findings of this study are available from the authors upon reasonable request. See Author contributions.

Received: 20 May 2018 Accepted: 14 March 2019

Published online: 23 April 2019

\section{References}

1. Coxib and traditional NSAID Trialists' (CNT) Collaboration. Vascular and upper gastrointestinal effects of non-steroidal anti-inflammatory drugs: metaanalyses of individual participant data from randomised trials. Lancet $\mathbf{3 8 2}$ 769-779 (2013).

2. Yellon, D. M. \& Hausenloy, D. J. Myocardial reperfusion injury. New Engl. J. Med. 357, 1121-1135 (2007).

3. Hausenloy, D. J. \& Yellon, D. M. Myocardial ischemia-reperfusion injury: a neglected therapeutic target. J. Clin. Investig. 123, 92-100 (2013).

4. Hausenloy, D. J. et al. Targeting reperfusion injury in patients with STsegment elevation myocardial infarction: trials and tribulations. Eur. Heart J. 38, 935-941 (2017).

5. Cheng, Y. et al. Cyclooxygenases, microsomal prostaglandin E synthase-1, and cardiovascular function. J. Clin. Investig. 116, 1391-1399 (2006).

6. Samuelsson, B., Morgenstern, R. \& Jakobsson, P. J. Membrane prostaglandin E synthase-1: a novel therapeutic target. Pharmacol. Rev. 59, 207-224 (2007).

7. Larsson, K. \& Jakobsson, P. J. Inhibition of microsomal prostaglandin E synthase-1 as targeted therapy in cancer treatment. Prostaglandins \& Other Lipid Mediat. 120, 161-165 (2015).

8. Jin, Y. et al. Pharmacodynamic comparison of LY3023703, a novel microsoma prostaglandin e synthase 1 inhibitor, with celecoxib. Clin. Pharmacol. Ther. 99, 274-284 (2016)

9. Tang, S. Y. et al. Cardiovascular consequences of prostanoid I receptor deletion in microsomal prostaglandin E synthase-1-deficient hyperlipidemic mice. Circulation 134, 328-338 (2016)

10. Wang, M. et al. Microsomal prostaglandin e2 synthase- 1 modulates the response to vascular injury. Circulation 123, 631-639 (2011).

11. Wang, M. et al. Deletion of microsomal prostaglandin E synthase-1 augments prostacyclin and retards atherogenesis. Proc. Natl Acad. Sci. USA 103, 14507-14512 (2006)

12. Hao, $\mathrm{H}$. et al. Protective role of mPGES-1 (microsomal prostaglandin $\mathrm{E}$ synthase-1)-derived PGE2 (prostaglandin E2) and the endothelial EP4 (prostaglandin $\mathrm{E}$ receptor) in vascular responses to injury. Arterioscler., Thromb., Vasc. Biol. 38, 1115-1124 (2018).

13. Camitta, M. G. et al. Cyclooxygenase-1 and -2 knockout mice demonstrate increased cardiac ischemia/reperfusion injury but are protected by acute preconditioning. Circulation 104, 2453-2458 (2001).

14. Guo, Y. et al. Evidence for an essential role of cyclooxygenase-2 as a mediator of the late phase of ischemic preconditioning in mice. Basic Res. Cardiol. 95, 479-484 (2000).

15. Shinmura, K. et al. Cyclooxygenase- 2 mediates the cardioprotective effects of the late phase of ischemic preconditioning in conscious rabbits. Proc. Natl Acad. Sci. USA 97, 10197-10202 (2000).

16. Xiao, C. Y. et al. Roles of prostaglandin I(2) and thromboxane A(2) in cardiac ischemia-reperfusion injury: a study using mice lacking their respective receptors. Circulation 104, 2210-2215 (2001).

17. Degousee, N. et al. Microsomal prostaglandin E2 synthase-1 deletion leads to adverse left ventricular remodeling after myocardial infarction. Circulation 117, 1701-1710 (2008).

18. Degousee, N. et al. Lack of microsomal prostaglandin E(2) synthase-1 in bone marrow-derived myeloid cells impairs left ventricular function and increases mortality after acute myocardial infarction. Circulation 125, 2904-2913 (2012).

19. Wu, D. et al. The effects of microsomal prostaglandin E synthase-1 deletion in acute cardiac ischemia in mice. Prostaglandins Leukot. Essent. Fat. Acids $\mathbf{8 1}$, 31-33 (2009).

20. Sugimoto, Y. \& Narumiya, S. Prostaglandin E receptors. J. Biol. Chem. 282, 11613-11617 (2007)

21. Xiao, C. Y. et al. Prostaglandin E2 protects the heart from ischemia-reperfusion injury via its receptor subtype EP4. Circulation 109, 2462-2468 (2004). 
22. Qian, J. Y. et al. Reduced cardiac remodeling and function in cardiac-specific EP4 receptor knockout mice with myocardial infarction. Hypertension 51, 560-566 (2008).

23. Zidar, N. et al. Cyclooxygenase in normal human tissues-is COX-1 really a constitutive isoform, and COX-2 an inducible isoform? J. Cell. Mol. Med. 13, 3753-3763 (2009).

24. Prasad, A., Stone, G. W., Holmes, D. R. \& Gersh, B. Reperfusion injury, microvascular dysfunction, and cardioprotection: the "dark side" of reperfusion. Circulation 120, 2105-2112 (2009).

25. Gutterman, D. D. et al. The human microcirculation: regulation of flow and beyond. Circ. Res. 118, 157-172 (2016).

26. Pries, A. R. \& Reglin, B. Coronary microcirculatory pathophysiology: can we afford it to remain a black box? Eur. Heart J. 38, 478-488 (2017).

27. Guerrero, M. D. et al. Synthesis and pharmacological evaluation of a selected library of new potential anti-inflammatory agents bearing the gammahydroxybutenolide scaffold: a new class of inhibitors of prostanoid production through the selective modulation of microsomal prostaglandin E synthase-1 expression. J. Med. Chem. 50, 2176-2184 (2007).

28. Woodward, D. F., Jones, R. L. \& Narumiya, S. International Union of Basic and Clinical Pharmacology. LXXXIII: classification of prostanoid receptors, updating 15 years of progress. Pharmacol. Rev. 63, 471-538 (2011)

29. Markovic, T., Jakopin, Z., Dolenc, M. S. \& Mlinaric-Rascan, I. Structural features of subtype-selective EP receptor modulators. Drug Discov. Today 22, 57-71 (2017)

30. Ito, H. et al. Clinical implications of the 'no reflow' phenomenon. A predictor of complications and left ventricular remodeling in reperfused anterior wall myocardial infarction. Circulation 93, 223-228 (1996).

31. van de Hoef, T. P. et al. Impact of coronary microvascular function on longterm cardiac mortality in patients with acute ST-segment-elevation myocardial infarction. Circ. Cardiovasc. Interv. 6, 207-215 (2013).

32. Koller, A. \& Kaley, G. Prostaglandins mediate arteriolar dilation to increased blood flow velocity in skeletal muscle microcirculation. Circ. Res. 67, 529-534 (1990).

33. Gwozdz, P., Drelicharz, L., Kozlovski, V. I. \& Chlopicki, S. Prostacyclin, but not nitric oxide, is the major mediator of acetylcholine-induced vasodilatation in the isolated mouse heart. Pharmacol. Rep.: PR 59, 545-552 (2007).

34. Foudi, N. et al. Differential reactivity of human mammary artery and saphenous vein to prostaglandin $\mathrm{E}(2)$ : implication for cardiovascular grafts. Br. J. Pharmacol. 163, 826-834 (2011).

35. Norel, X., de Montpreville, V. \& Brink, C. Vasoconstriction induced by activation of EP1 and EP3 receptors in human lung: effects of ONO-AE-248, ONO-DI-004, ONO-8711 or ONO-8713. Prostaglandins \& Other Lipid Mediat. 74, 101-112 (2004).

36. Ozen, G. et al. Inhibition of microsomal PGE synthase-1 reduces human vascular tone by increasing PGI2: a safer alternative to COX-2 inhibition. Br. J. Pharmacol., https://doi.org/10.1111/bph.13939 (2017)

37. Duffin, R. et al. Prostaglandin E(2) constrains systemic inflammation through an innate lymphoid cell-IL-22 axis. Science 351, 1333-1338 (2016).

38. Timmers, L. et al. Cyclooxygenase- 2 inhibition increases mortality, enhances left ventricular remodeling, and impairs systolic function after myocardial infarction in the pig. Circulation 115, 326-332 (2007).

39. Schmidt, M. et al. Cardiovascular safety of non-aspirin non-steroidal antiinflammatory drugs: review and position paper by the working group for Cardiovascular Pharmacotherapy of the European Society of Cardiology. Eur. Heart J. 37, 1015-1023 (2016).

40. Grosser, T., Ricciotti, E. \& FitzGerald, G. A. The cardiovascular pharmacology of nonsteroidal anti-inflammatory drugs. Trends Pharmacol. Sci. 38, 733-748 (2017).

41. Gao, E. et al. A novel and efficient model of coronary artery ligation and myocardial infarction in the mouse. Circ. Res. 107, 1445-1453 (2010).

42. Safholm, J. et al. PGE2 maintains the tone of the guinea pig trachea through a balance between activation of contractile EP1 receptors and relaxant EP2 receptors. Br. J. Pharmacol. 168, 794-806 (2013).

43. af Forselles, K. J. et al. In vitro and in vivo characterization of PF-04418948, a novel, potent and selective prostaglandin $\mathrm{EP}(2)$ receptor antagonist. Br. J. Pharmacol. 164, 1847-1856 (2011)

44. Juteau, H. et al. Structure-activity relationship of cinnamic acylsulfonamide analogues on the human EP3 prostanoid receptor. Bioorg. \& Med. Chem. 9, 1977-1984 (2001).

45. Wilson, R. J. et al. GW627368X ((N-\{2-[4-(4,9-diethoxy-1-oxo-1,3-dihydro2H-benzo[f]isoindol-2-yl)phenyl]acetyl $\}$ benzene sulphonamide): a novel, potent and selective prostanoid EP4 receptor antagonist. Br. J. Pharmacol. 148, 326-339 (2006).

46. Hao, H. F. et al. Rhynchophylline ameliorates endothelial dysfunction via Src-PI3K/Akt-eNOS cascade in the cultured intrarenal arteries of spontaneous hypertensive rats. Front. Physiol. 8, 928 (2017).

47. Zhang, X., Yang, N., Ai, D. \& Zhu, Y. Systematic metabolomic analysis of eicosanoids after omega-3 polyunsaturated fatty acid supplementation by a highly specific liquid chromatography-tandem mass spectrometry-based method. J. Proteome Res. 14, 1843-1853 (2015).

48. Song, W. L., Lawson, J. A., Wang, M., Zou, H. \& FitzGerald, G. A. Noninvasive assessment of the role of cyclooxygenases in cardiovascular health: a detailed HPLC/MS/MS method. Methods Enzymol. 433, 51-72 (2007).

49. Galaup, A. et al. Protection against myocardial infarction and no-reflow through preservation of vascular integrity by angiopoietin-like 4. Circulation 125, 140-149 (2012).

\section{Acknowledgements}

We thank Yuuki Nagao from ONO Pharmaceutical Co. Ltd. for supplying us the research compounds. We thank Sheng Hu from Fuwai Hospital for mouse breeding and colony maintenance. This work was supported by the National Natural Science Foundation of China (81370222 and 81570269), Chinese Academy of Medical Sciences Innovation Fund for Medical Sciences (2017-I2M-1-008, 2016-I2M-1-003/006), a research award from the National 1000-Talent Plan of China, and research funds from Fuwai Hospital, Peking Union Medical College and Chinese Academy of Medical Sciences. Dr. FitzGerald is the McNeil Chair of Translational Medicine and Therapeutics and received support from NHLBI (HL117798).

\section{Author contributions}

L.Z., C.X., and X.H., with equal contribution, performed most of the experiments, and analyzed the data. L.Z. and C.X. wrote the methods. H.H. contributed to the cardiac flow measurement, the vascular ring experiment and the relevant method writing. Q.W. and H.C. assisted in animal study, sample collection and analysis. X.Z. measured prostanoids and their metabolites. R.B., Y.H., X.C., D.L., and G.F. provided advises on the study and critically read the manuscript. R.B. and G.F. provided mice for this study. Y.H. provided study support on the vascular ring experiments. M.W. conceived and supervised the study, and wrote the manuscript.

\section{Additional information}

Supplementary Information accompanies this paper at https://doi.org/10.1038/s41467 019-09492-4.

Competing interests: The authors declare no competing interests.

Reprints and permission information is available online at http://npg.nature.com/ reprintsandpermissions/

Journal peer review information: Nature Communications thanks Barry Rubin and the other anonymous reviewers for their contribution to the peer review of this work.

Publisher's note: Springer Nature remains neutral with regard to jurisdictional claims in published maps and institutional affiliations.

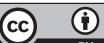

Open Access This article is licensed under a Creative Commons Attribution 4.0 International License, which permits use, sharing adaptation, distribution and reproduction in any medium or format, as long as you give appropriate credit to the original author(s) and the source, provide a link to the Creative Commons license, and indicate if changes were made. The images or other third party material in this article are included in the article's Creative Commons license, unless indicated otherwise in a credit line to the material. If material is not included in the article's Creative Commons license and your intended use is not permitted by statutory regulation or exceeds the permitted use, you will need to obtain permission directly from the copyright holder. To view a copy of this license, visit http://creativecommons.org/ licenses/by/4.0\%

(C) The Author(s) 2019 